\title{
Cigarette smoke exposure enhances transforming acidic coiled-coil-containing protein 2 turnover and thereby promotes emphysema
}

\author{
Rama K. Mallampalli, ${ }^{1}$ Xiuying Li, ${ }^{2,3}$ Jun-Ho Jang, ${ }^{2}$ Tomasz Kaminski, ${ }^{4}$ Aki Hoji, ${ }^{2}$ Tiffany Coon, ${ }^{2}$ \\ Divay Chandra, ${ }^{2}$ Starr Welty, ${ }^{5,6}$ Yaqun Teng, ${ }^{7}$ John Sembrat, ${ }^{2}$ Mauricio Rojas, ${ }^{2}$ Yutong Zhao, ${ }^{8}$ \\ Robert Lafyatis, ${ }^{2}$ Chunbin Zou, ${ }^{2,3}$ Frank Sciurba, ${ }^{2}$ Prithu Sundd, ${ }^{4}$ Li Lan, ${ }^{9}$ and Toru Nyunoya ${ }^{2,3}$ \\ 'Department of Medicine, The Ohio State University College of Medicine, Columbus, Ohio, USA . 2Department of Medicine, \\ University of Pittsburgh, Pittsburgh, Pennsylvania, USA. ${ }^{3}$ Medical Specialty Service Line, Veterans Affairs Pittsburgh \\ Healthcare System, Pittsburg, Pennsylvania, USA. ${ }^{4}$ Vascular Medical Institute, University of Pittsburgh, Pittsburgh, \\ Pennsylvania, USA. ${ }^{5}$ Department of Microbiology \& Molecular Genetics, University of Pittsburgh, Pittsburgh, Pennsylvania, \\ USA. 'UMPC Hillman Cancer Center, Pittsburgh, Pennsylvania, USA. 'School of Medicine, Tsinghua University, No. 1 \\ Tsinghua Yuan, Beijing, China. ${ }^{8}$ Department of Physiology and Cell Biology, The Ohio State University College of Medicine, \\ Columbus, Ohio, USA. ${ }^{9}$ Department of Radiation Oncology, Massachusetts General Hospital Cancer Center, Harvard \\ Medical School, Charlestown, Massachusetts, USA.
}

Our integrative genomic and functional analysis identified transforming acidic coiled-coilcontaining protein 2 (TACC2) as a chronic obstructive pulmonary disease (COPD) candidate gene. Here, we found that smokers with COPD exhibit a marked decrease in lung TACC2 protein levels relative to smokers without COPD. Single cell RNA sequencing reveals that TACC2 is expressed primarily in lung epithelial cells in normal human lungs. Furthermore, suppression of TACC2 expression impairs the efficiency of homologous recombination repair and augments spontaneous and cigarette smoke extract-induced (CSE-induced) DNA damage and cytotoxicity in immortalized human bronchial epithelial cells. By contrast, enforced expression of TACC2 attenuates the CSE effects. We also found that CSE enhances TACC2 degradation via the ubiquitin-proteasome system mediated by the ubiquitin E3 ligase subunit, F box L7. Furthermore, cellularly expressed TACC2 proteins harboring naturally occurring mutations exhibited altered protein lifespan coupled with modified DNA damage repair and cytotoxic responses. CS triggers emphysematous changes accompanied by accumulated DNA damage, apoptosis of alveolar epithelia, and lung inflammation in Tacc2 $^{-/-}$compared with Tacc2 $^{+/+}$mice. Our results suggest that CS destabilizes TACC2 protein in lung epithelia by the ubiquitin proteasome system, leading to subsequent DNA damage, cytotoxicity, and emphysema.

Authorship note: RKM and XL contributed equally to this work.

Conflict of interest: The authors have declared that no conflict of interest exists.

Copyright: (c) 2020, American Society for Clinical Investigation.

Submitted: October 30, 2018 Accepted: December 18, 2019 Published: January 30, 2020.

Reference information: JCI Insight. 2020;5(2):e125895.

https://doi.org/10.1172/jci.

insight.125895.

\section{Introduction}

Cigarette smoking is the major risk factor for chronic obstructive pulmonary disease (COPD), and an accumulating dose over 40 pack-years is a major predictable factor (1). Unfortunately, mortality due to COPD has been increasing, and it recently has emerged as the third leading cause of death in the United States (2). Despite the growing public threat from COPD, there has been no effective therapy that significantly reduces the burden of COPD. Insights into COPD pathogenesis suggest a role of persistent DNA damage in the pathobiology of COPD $(3,4)$. In response to cigarette smoke (CS) exposure, lung cells exhibit an orchestrated signaling process, the DNA damage response (DDR), which senses DNA damage and initiates DNA repair to maintain genomic integrity (5). The DDR signaling is mediated by phosphoinositide 3-kinase related protein kinases (PIKKs), including ataxia telangiectasia mutated (ATM). ATM, primarily activated by double-strand breaks (DSBs), regulates cell cycle progression and DNA repair through phosphorylating multiple key substrates, including histone H2A at serine $139(\gamma \mathrm{H} 2 \mathrm{AX})$. There are 2 major DSB repair pathways, nonhomologous end-joining (NHEJ) and homologous recombination (HR) (6). 
NHEJ repairs DSBs by rejoining DNA without the use of extensive homology. In contrast, HR utilizes homology between the sequences of damaged and undamaged DNA strands. A human study demonstrated that the lungs of smokers with COPD exhibit DNA damage accumulation accompanied by increased $\gamma \mathrm{H} 2 \mathrm{AX}$ relative to those of smokers without COPD (4). DNA damage may account for apoptosis, inflammation, and cellular senescence in lung epithelial cells (LECs) in COPD patients (7). In fact, a previous in vivo mouse study demonstrated that activation of the ATM/p53/p21 contributes to the development of CS-induced emphysema (8). However, the molecular inputs to this pathway responsible for persistent DNA damage remain to be elucidated.

Our recent human studies using both genomic and functional analyses identified TACC2 as a COPD candidate gene (9). Whole exome sequencing (WES) among 62 smokers with severe COPD and 30 resistant smokers identified 7 rare deleterious variants of TACC2 that cause nonsense or nonsynonymous mutations in 8 COPD subjects (12.9\%), in contrast to none in resistant smokers (9). Furthermore, suppression of TACC2 by siRNA transfection markedly enhanced CS-induced apoptotic cell death in cultured immortalized human bronchoepithelial cells (HBECs) (9). Interestingly, a large database from the genome-wide association study (GWAS) performed on about 450,000 United Kingdom Biobank (UK Biobank) White British individuals revealed several nonsynonymous TACC2 mutations potentially linked to emphysema (http://geneatlas.roslin.ed.ac.uk).

The TACC2 protein is a member of the transforming acidic coiled-coil (TACC) family that regulates microtubule homeostasis (10). TACCs are expressed as Drosophila TACC (D-TACC) in flies, whereas TACC1, TACC2, and TACC3 are seen in mammals. The TACC family possesses a highly conserved C-terminal TACC domain that may regulate versatile functions, including genomic stability, transcription, protein trafficking, and cytoskeleton organization (11). In a fly model, the protein levels of D-TACC are tightly regulated. Altered levels or dysfunction of D-TACC2 causes spindle dysfunction and mitotic defects, often resulting in early embryonic death $(12,13)$. In humans, all TACC proteins are present in the centrosome to regulate microtubule organization, but they exhibit some distinction in temporal expression. TACC2 is highly present in the centrosome throughout the cell cycle, whereas both TACC1 and TACC3 are localized to the centrosome only during mitosis. Human TACC2 has 2 major transcripts: 4.2 $\mathrm{kb}$ and $9.7 \mathrm{~kb}$ mRNAs. In adult tissues, the $4.2 \mathrm{~kb}$ transcript is more abundantly expressed in brain, prostate, thyroid, and airways (14). TACC2 mutations and dysregulated protein expression is associated with human malignancies, including breast and ovarian cancers, suggesting a potential role of TACC in regulating genomic stability and carcinogenesis $(15,16)$. Tacc $2^{-/-}$mice are viable and grow normally without a spontaneous clinical phenotype (17). However, the role of TACC2 in stress responses such as CS-induced injury, cytotoxicity, or its mechanistic link to disorders such as emphysema is unknown.

Ubiquitination is an important posttranslational modification that maintains proteostasis and regulates diverse processes through branding proteins for degradation either by the proteasome or lysosome (18). Ubiquitin is bound to a target protein through a series of enzymatic reactions involving an E1, an E2, and an E3 enzyme, corresponding to the 3 sequential steps - ubiquitin activation, conjugation, and ligation, respectively (18). The Skp1-Cullin-F-box (SCF) ubiquitin ligase complex belongs to the most studied E3 family (19) containing an adaptor receptor subunit, termed F-box protein, that targets hundreds of substrates (20). The ability of F-box proteins to engage their targets often requires a posttranslational modification within the target that enhances E3 ligase recruitment to optimize ubiquitination. The ubiquitin proteasome system (UPS) is activated in COPD, with accumulation of ubiquitinated proteins in the lungs of severe COPD subjects (21).

In this study, we show that TACC2 is depleted in the lungs of individuals with severe COPD. We found that TACC2, expressed primarily in LECs, plays an important role in DNA repair in response to CS-induced epithelial damage. CS exposure enhances TACC2 degradation mediated by another centrosome protein, F-box protein Fbx17, that targets TACC2 for cellular disposal. These observations provide molecular insight into a unique pathway, whereby CS triggers DNA damage and apoptosis in this chronic illness.

\section{Results}

Smokers with COPD exhibit decreased pulmonary TACC2 protein. Our genomic and functional studies identified TACC2 as a COPD candidate gene (9). However, TACC2 protein levels in the lungs of patients with COPD are unknown. To minimize potential effects from recent CS exposure, we selected study subjects who stopped smoking for at least 6 months at different stages of COPD severity (Table 1). Lung tissues from smokers with COPD (Global Initiative for Obstructive Lung Disease [GOLD] stage $2[n=6]$ and stage 3 or $4[n=10])$ were evaluated and compared with smokers with normal lung 
Table 1. Demographic data

\begin{tabular}{|c|c|c|c|c|}
\hline & Control $(n=6)$ & $\operatorname{GOLD2}(n=6)$ & GOLD3-4 $(n=10)$ & $P$ value \\
\hline Age & $70.3 \pm 5.1$ & $70.3 \pm 7.8$ & $58.7 \pm 7.3$ & 0.013 \\
\hline Sex & $2 \mathrm{M} / 4 \mathrm{~F}$ & $1 \mathrm{M} / 5 \mathrm{~F}$ & $5 \mathrm{M} / 5 \mathrm{~F}$ & 0.47 \\
\hline $\mathrm{FEV}_{1} / \mathrm{FVC}$ & $0.78 \pm 0.04$ & $0.58 \pm 0.09$ & $0.29 \pm 0.05$ & $<0.001$ \\
\hline Percent FEV & $91.8 \pm 14.0$ & $70.2 \pm 6.7$ & $23.7 \pm 7.2$ & $<0.001$ \\
\hline Percent DLCO & $86.3 \pm 8.1$ & $78.5 \pm 18.1$ & $24.4 \pm 6.6$ & 0.002 \\
\hline
\end{tabular}

The stage of COPD was determined by the Global Initiative for Obstructive Lung Disease (GOLD) criteria (42). Stage 2, moderate; Stage 3, severe; and stage 4 , very severe. Control represents smokers with normal pulmonary function. Data are expressed as mean $\pm \mathrm{SD}$. An overall ^ $P$ value is based on the KrusalWallis test. FEV ${ }_{1}$, forced expiratory volume in 1 second; FVC, forced vital capacity; DLCO, the diffusing capacity of the lungs for carbon monoxide.

function $(n=6)$. TACC2 protein levels were markedly depleted in the lungs of smokers with moderately severe or very severe COPD as compared with smokers without COPD (Figure 1, A and B). By contrast, mRNA levels of TACC2 were not significantly altered in the lungs of smokers with COPD when compared with smokers without COPD (Figure 1C). These data suggest that pulmonary levels of TACC2 protein are decreased by a posttranscriptional mechanism in subjects with COPD. We also evaluated TACC2 protein levels in the lungs of nonsmoking and actively smoking subjects without known lung disease ( $n=4$, each group). TACC2 protein is present in the lungs of nonsmoking subjects but is decreased in the lungs of active smokers (Supplemental Figure 1A; supplemental material available online with this article; https://doi.org/10.1172/jci.insight.125895DS1).

Next, to determine the cell specificity of TACC2 expression, single cell mRNA sequencing was conducted using 3 normal human subjects. Two-dimensional T-distributed stochastic neighbor embedding (t-SNE) plots were generated based on global gene expression relationships among 9987 total cells (Figure 1D). Cells broadly clustered into 13 groups. TACC2 and 3 marker genes for LECs, including FOXJ1 (ciliated airway epithelial cells), SFTPC (alveolar type 2 epithelial cells), and EPCAM(LECs), are shown with level of expression indicated by intensity of purple. The data suggest that TACC2 is primarily expressed in both alveolar and bronchial epithelial cells in normal human lungs. We also performed scRNA sequencing to examine TACC2 expression in the lung of severe COPD subjects $(n=3)$. There was no significant change in TACC2 gene expression in LECs between normal and COPD subjects (Supplemental Figure 1B).

$T A C C 2$ is required to protect against CS-induced DNA damage and cytotoxicity. To determine the effects of CS on DDR/repair proteins in primary LECs, both primary HBECs and primary human alveolar epithelial cells (pHAECs) were cultured with or without $\%$ CSE for 24 hours. CSE increased phosphorylation of ATM (ser1981) and H2AX (ser139) without altering the individual total protein levels in both primary cell cultures (Figure 2A). Next, to determine the role of TACC2 protein in CSE-induced DNA damage and cytotoxicity, HBEC2 cells (immortalized by stable transduction with TERT, a catalytic subunit of telomerase and cyclin-dependent kinase 4; ref. 22) were transfected with siRNA for 48 hours and further cultured with or without $2 \%$ CSE for 24 hours. siTACC2 decreased TACC2 protein by $60 \%$ coupled with increased levels of $\gamma \mathrm{H} 2 \mathrm{AX}$ (but not phosphorylated ATM; pATM), the latter even in the absence of CSE (Figure 2B). CSE-induced DDRs and cytotoxicity were further augmented by TACC2 protein depletion (Figure 2, B and C). To further determine the function of TACC2, BEAS-2B cells were transfected with double nickase plasmids. Among more than 20 clones isolated from the transfected cells, we confirmed that 1 clone (clone \#3) showed a marked reduction of TACC2 protein expression (Figure 2D), suggesting successful $\mathrm{KO}$ of TACC2. TACC2 CRISPR cell lines grew very slowly compared with a control cell line (data not shown). Flow cytometric analysis (TACC2 clone \#3 and control cells) showed a marked increase in spontaneous DNA damage $(\gamma \mathrm{H} 2 \mathrm{AX})$ and apoptosis (positive annexin V) (Figure 2E). We also generated a V5-tagged TACC2 plasmid (pcDNA3.1). TACC2 overexpression attenuated levels of CSE-induced DNA damage markers (pATM and $\gamma \mathrm{H} 2 \mathrm{AX}$ ) and cytotoxicity (Figure 2, F and G).

TACC 2 is partially translocated into the nucleus and associated with $\gamma H 2 A X$ in response to CS. To determine nuclear translocation of TACC2, the subcellular fractionation of HBEC2 cells exposed to CSE was carried out. CSE exposure slightly decreased TACC2 expression in the cytosol but increased TACC2 in the nucleus (Figure 3A). These results suggest that CSE exposure alters TACC2 subcellular localization with 
A
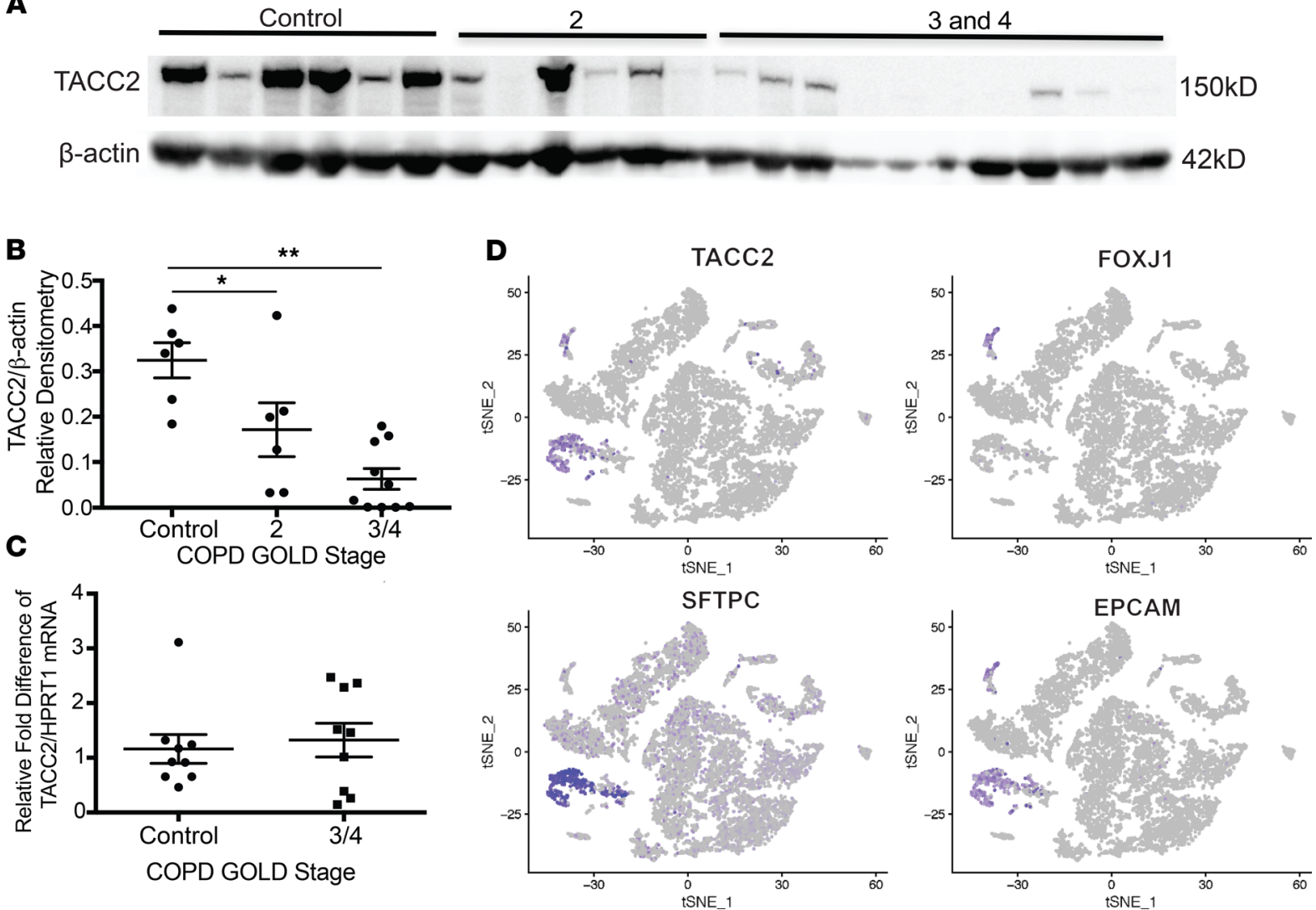

Figure 1. Smokers with COPD exhibit decreased TACC2 protein. (A) The stage of COPD was determined by the Global Initiative for Obstructive Lung Disease (COLD) criteria (44). Stage 2, moderate; stage 3, severe; and stage 4, very severe. Control represents smokers with normal pulmonary function. Whole lung parenchyma lysates were obtained from a total of 22 smokers with various GOLD stages of COPD. Immunoblot (IB) analysis was performed for TACC2. (B) The densitometry data (TACC2/ $\beta$-actin) obtained from $\mathbf{A}$ are expressed as mean \pm SEM. One-way ANOVA with Bonferroni correction was made. ${ }^{*} P<0.05$ (control vs. GOLD stage 2 ); ${ }^{* *} P<0.01$ (control vs. GOLD stage 3/4). (C) Total RNA was isolated from whole lung parenchymal tissues obtained from the same donors (control and GOLD stages 3 and 4) as in A. Steady-state levels of TACC2 mRNA were measured by RT-PCR. The relative fold difference compared with HPRT1 (control) was expressed. Data are expressed as mean \pm SEM. (D) Single cell RNA sequencing was conducted using lung parenchymal tissues obtained from 3 normal human subjects. t-SNE blots were shown. The intensity of purple indicates levels of gene expression. FOXJ1, Forkhead Box J1; SFTPC, Surfactant protein C; EPCAM, Epithelial cell adhesion molecule.

a potential shift toward the nucleus. Furthermore, to determine if TACC2 localizes to DNA damage sites, co-IP with TACC2 antibody was performed in cell lysates harvested from CSE-exposed or -unexposed HBEC2 cells. We found that CSE increased physical interaction between TACC2 and $\gamma \mathrm{H} 2 \mathrm{AX}$ after CSE exposure (Figure 3B). Nuclear TACC2 formed small foci that at least partially colocalized with $\gamma \mathrm{H} 2 \mathrm{AX}$ foci (Figure 3B, arrows). Here, increased $\gamma \mathrm{H} 2 \mathrm{AX}$ levels without TACC2 and colocalization of TACC2 with $\gamma \mathrm{H} 2 \mathrm{AX}$ suggest that TACC2 might be involved in the clearance of DSB, which are induced by CSE. Both HR and NHEJ are major pathways for the repair of DSBs in mammalian cells. To further support a purported DSB repair function of TACC2, both HR and NHEJ reporter assays were performed. In these assays, cleavage of the I-SceI sites (resulting in DSBs) in DR-GFP and Ej5-GFP sequences induces HR and NHEJ, respectively. For HR assays, DR-GFP is composed of 2 differentially mutated green fluorescent protein (GFP) genes oriented as direct repeats (DR); the upstream repeat contains the recognition site for the rare-cutting I-SceI endonuclease, and the downstream repeat is a $5^{\prime}$ and $3^{\prime}$ truncated GFP fragment. Transient expression of I-SceI leads to a DSB in the upstream GFP gene; an active HR process to repair the DSB results in $\mathrm{GFP}^{+}$cells, which are quantified by flow cytometry. For the NHEJ assays, a reporter (EJ5-GFP) was used to measure end joining (EJ) between distal DSB ends of 2 tandem I-SceI sites. These distal-EJ events do not require access to homology and, thus, are distinct from the repair events described above. Indeed, this assay provides a measure of DSB end protection during EJ via physical analysis of distal-EJ products to determine the frequency of I-SceI restoration. Thus, cleavage of the I-SceI sites (resulting in DSBs) in DR-GFP and EJ5-GFP sequences induces HR and NHEJ, respectively. 
A

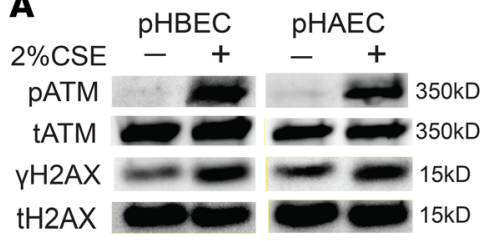

B

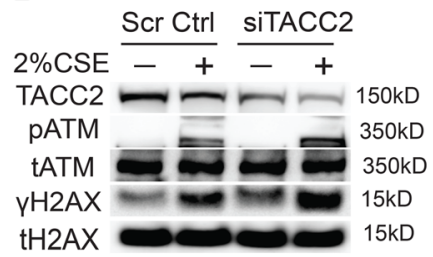

C

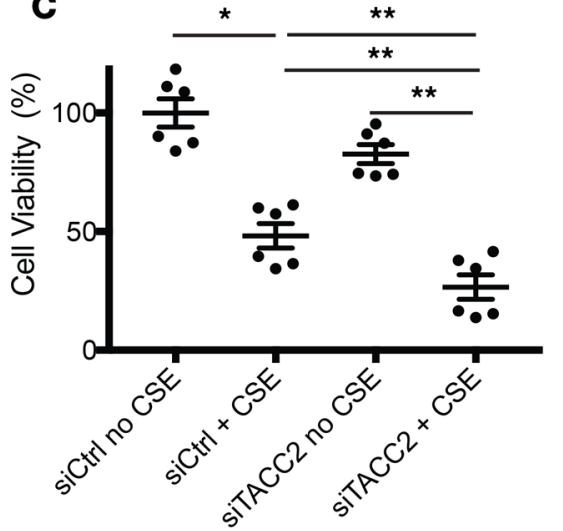

D

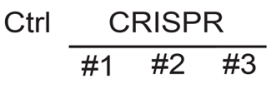

TACC2

GAPDH
E
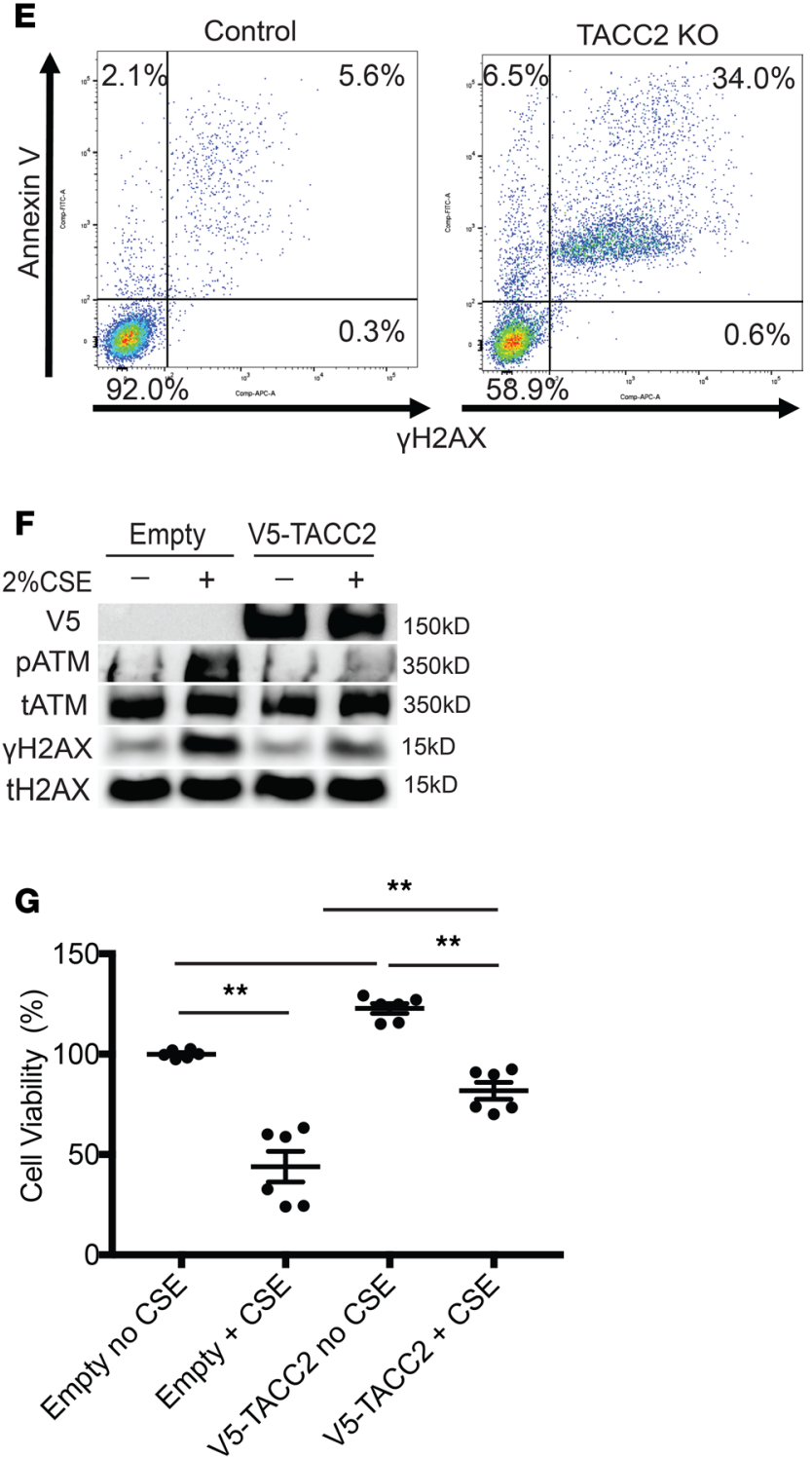

Figure 2. TACC2 is required to protect against cigarette smoke-induced DNA damage and cytotoxicity. (A) pHBECs and pHAECs were cultured with or without $2 \%$ CSE for 24 hours. IB analysis was performed for PATM and $\gamma$ H2AX. (B) HBEC2 cells transfected with siTACC2 or a scrambled control RNA (Scr Ctrl) were cultured with or without $2 \%$ CSE for 24 hours. IB analysis was performed for TACC2, pATM, and $\gamma H 2 A X$. IB data are representative of 3 independent experiments. (C) HBEC2 cells were treated as in B. Cell viability was determined by the (3-[4, 5-dimethylthiazolyl-2]-2, 5-diphenyltetrazolium bromide) (MTT) assay. Data are expressed as mean \pm SEM for 2 independent experiments with triplicated samples. One-way ANOVA with Bonferroni correction was made. (D) BEAS-2B cells were transfected with the double nickase plasmid targeting TACC2, and 3 colonies were selected. IB analysis was performed for TACC2. IB data are representative of 3 independent experiments. (E) A flow cytometric assay with dual staining of annexin $V$ and $\gamma \mathrm{H} 2 \mathrm{AX}$ was performed for TACC2 CRISPR clone \#3 and controlled cells. (F) BEAS-2B cells transfected with V5-tagged TACC2 plasmid were incubated with or without $2 \%$ CSE for 24 hours for 2 independent experiments with triplicate samples. Immunoblot analysis was performed for V5, pATM, and $\gamma$ H2AX. IB data are representative of 3 independent experiments. (G) The cells were treated as in F. Cell viability was determined by the MTT assay. Data are expressed as mean \pm SEM for 2 independent experiments with triplicate samples. One-way ANOVA with Bonferroni correction was conducted.

Repair efficiency was monitored as the percentage of cells expressing fully repaired GFP. Indeed, causally, TACC2 depletion significantly decreased the efficiency of HR repair (Figure 3C) but not NHEJ repair (data not shown). Thus, functionally, TACC2 appears to play a crucial role in spontaneous and CSE-induced DDR and repair.

CS decreases TACC2 protein in human LECs. To determine the mechanism of CSE on TACC2 protein depletion, we cultured immortalized HBECs (HBEC2 and BEAS-2B cells) in the absence or presence of CSE (1\%, $2 \%, 4 \%$, or $6 \%$ ) for 24 hours. CSE decreased TACC2 protein levels in a concentration-dependent manner (Figure 4, A and B). Likewise, CSE decreased TACC2 in a human epithelial cell line, A549 (Figure 4C). 
Furthermore, we verified the CSE effects on TACC2 in primary LECs, pHBECs, and pHAECs isolated from 3 and 1 nonsmoking donor, respectively (Figure 4D). Our in vitro studies demonstrated that up to $2 \%$ CSE does not cause significant apoptotic cell death in cultured HBEC2 cells (9). We next determined the kinetics of effects of CS on TACC2 cellular concentrations for various time periods up to 24 hours. CSE at $2 \%$ significantly triggered reduction of immunoreactive TACC2 at 8 hours, an effect that progressively decreased to 24 hours (Figure 4E). However, TACC2 mRNA levels were not significantly altered (Figure 4F). We next determined the effects of CSE on TACC2 protein lifespan using the protein synthesis inhibitor cycloheximide (CHX). The half-life of TACC2 was longer than 24 hours in the absence of CSE but decreased to 8 hours after CSE exposure (Figure 4G). Thus, CSE decreases TACC2 protein lifespan in lung epithelia via a posttranslational mechanism.

An E3 ligase F-box component targets TACC2 for polyubiquitination. To determine the subcellular pathway for CSE-induced rapid degradation of TACC2 protein, we cultured 2\% CSE-exposed HBEC2 cells with the proteasome inhibitor MG132 or the lysosome inhibitor leupeptin for 8 hours in the presence of CHX. Addition of MG132 to the cells led to no decrease in TACC2 protein, whereas this was not observed in the absence of this compound or with leupeptin (Figure 5A); this suggests that TACC2 protein is degraded by CSE via the UPS. To determine if ubiquitination is sufficient to promote TACC2 degradation, we overexpressed hemagglutinin-tagged (HA-tagged) ubiquitin in BEAS-2B cells. Ubiquitin overexpression enhanced the degradation of TACC2 in a concentration-dependent manner (Figure 5B). To confirm that ubiquitination of TACC2 is increased by CSE, we cultured BEAS-2B cells with $2 \%$ CSE in the presence of MG132 for 2 hours and performed IP with TACC2 antibody. Immunoblot analysis of the precipitates by TACC2 antibody demonstrated that polyubiquitination of endogenous TACC2 was enhanced by CSE (Figure 5C). Next, given the fact that FBXL7, an F-box protein of the E3 ligase subunit, is localized in the centrosome, we hypothesized that FBXL7 contributes to TACC2 degradation. To further evaluate this, cells were transfected with increasing concentrations of V5-tagged Fbxl7 or Fbxl8 plasmid. TACC2 expression was selectively decreased in a dose-dependent manner by Fbxl7 (Figure 5D). To verify if FBXL7 associates and targets TACC2, co-IP with TACC2 antibody was performed in cell lysates harvested from CSE-exposed or -unexposed BEAS-2B cells. We found that FBXL7 interacts with TACC2, an effect augmented after CSE exposure (Figure 5E). To further validate physical association and degradation of TACC2 with FBXL7, in vitro ubiqutination assays were performed. The ubiquitin E3 ligase $\mathrm{SCF}^{\mathrm{FBX} 17}$ sufficiently catalyzed TACC2 polyubiquination (Figure $5 \mathrm{~F}$ ).

SCF-based F-box proteins are recruited to their substrates by molecular recognition signals such as phosphorylation sites. Mass spectrometry-based proteomic analysis in response to DNA damage reveals several consensus phosphorylation sites within TACC2 (23). To determine the effects of the site-specific TACC2 phosphorylations on the physical interaction with FBXL7, BEAS-2B cells were transfected with V5-tagged TACC2 WT and phosphorylation-defective mutant (T151A, S304A, S395A, and S399A) plasmids for 48 hours. Cells were further incubated with 2\% CSE for 4 hours. Among these mutants, expression of both S304A and S399A TACC2 variants decreased the physical interaction with FBXL7 (Figure 5G). These results suggest that specific molecular sites within TACC2 serve as recruitment signals for the E3 ligase subunit to potentially modulate DNA repair.

Naturally occurring mutations and targeted deletion of TACC2 alter functional and phenotypic responses to CS. We selected several rare nonsynonymous mutations of TACC2 potentially linked to the diagnosis of emphysema from the UK Biobank data (Figure 6A). To determine the lifespan of the individual TACC2 mutants in response to CSE, BEAS-2B cells were transfected with various plasmids encoding the individual TACC2 mutants for 48 hours, followed by incubation with or without CSE for up to 24 hours. The half-lives of individual TACC2 mutants were not different relative to the WT for up to 24 hours in the absence of CSE (data not shown). However, in the presence of CSE, the half-life of the G808R mutant was longer ( 24 hours) and that of the T560M mutant was shorter ( $\sim 4$ hours) than WT, E131, and T269M mutants ( $\sim 8$ hours) (Figure $6 \mathrm{~B})$. Next, to determine the type of TACC2 polyubiquitination, we cultured BEAS-2B cells with $2 \%$ CSE in the presence of MG132 for 2 hours and performed co-IP with TACC2 antibody. Immunoblot analysis of the precipitates using K48 and K63 antibodies demonstrated that CSE-induced TACC2 polyubiquitination was formed by the K48 but not the K63-specific linkages (Figure 6C). To determine the effects of TACC2 mutants on CSE-induced polyubiquitination, BEAS-2B cells were transfected with V5-tagged TACC2 WT and mutant (T560M and G808R) plasmids for 48 hours, followed by CSE for 2 hours, and samples were processed for IP with V5 antibody. K48-specific polyubiquitination by CSE was increased in the T560M mutant 

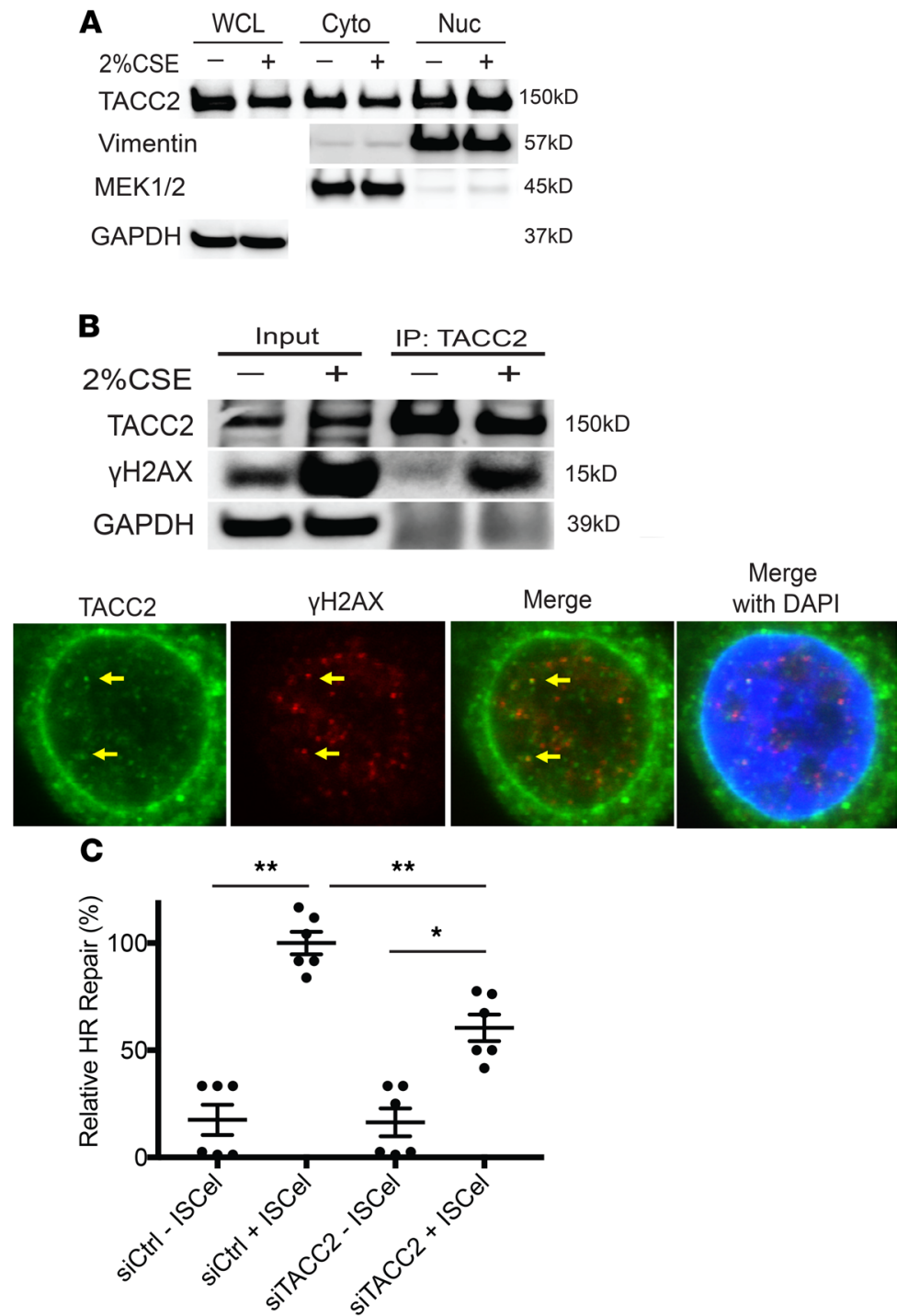

Figure 3. TACC2 is partially translocated into the nucleus and associated with $\gamma \mathrm{H} 2 \mathrm{AX}$ in response to cigarette smoke. (A) Subcellular localization of TACC2 protein in HBEC2 cells exposed to $2 \%$ CSE for 2 hours. WCL, whole cell lysates; Cyto, cytoplasm; Nuc, nucleus. IB data are representative of 3 independent experiments. (B) IP was performed using primary TACC2 antibody in cell lysates harvested from CSE-exposed or -unexposed HBEC2 cells. IB analysis was performed using $\gamma \mathrm{H} 2 \mathrm{AX}$ antibody. IB data are representative of 3 independent experiments. Immunofluorescence (IF) analysis of TACC2 and $\gamma \mathrm{H} 2 \mathrm{AX}$ coexpression in CSE-exposed HBEC2 cells. A representative micrograph showing TACC2-(green), $\gamma \mathrm{H} 2 \mathrm{AX}$ (red), and DAPI (blue). Original magnification, 600×. (C) DR-GFP-stably expressing cells were transfected with siTACC2, followed by transfection with pCMV-IScel plasmid. The GFP' cell fraction was measured by a FACS, and data are shown graphically. Data are expressed as mean \pm SEM for 2 independent experiments with triplicate samples. One-way ANOVA with Bonferroni correction was conducted $\left({ }^{*} P<0.05 ;{ }^{* *} P<0.01\right)$.

and decreased in the G808R mutant relative to WT (Figure 6D). Furthermore, the physical association between T560M with FBXL7 was increased in the T560M mutant and decreased with the G808R mutant relative to WT (Figure $6 \mathrm{E}$ ). To determine the function of these TACC2 mutants, BEAS-2B cells transfected with individual TACC2 plasmids were cultured with or without CSE for 48 hours. Cellular expression of T269M and T560M mutants resulted in modestly decreased cell viability in CSE-exposed cells relative to WT and in substantially lower viability compared with non-CSE exposed conditions. In contrast, expression of the G808R mutant increased the cell viability after CSE exposure (Figure 6F). DNA damage markers (pATM and $\gamma \mathrm{H} 2 \mathrm{AX}$ ) were also increased in the E131, T269M, T560M mutants, but they were decreased in the G808R mutant (Figure 6G). These results suggest that nonsynonymous mutations of TACC2 modulate protein stability by the UPS and impact cell viability and DNA damage after tobacco smoke exposure. 
A

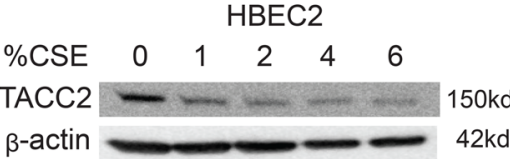

B

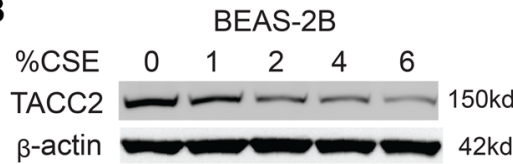

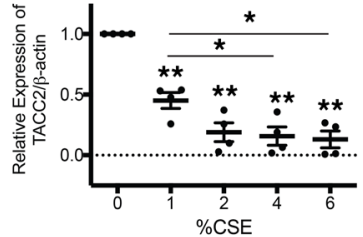

C
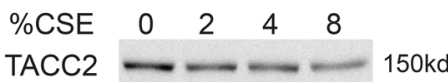

$\beta$-actin

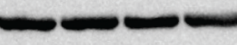

$42 \mathrm{kd}$
D

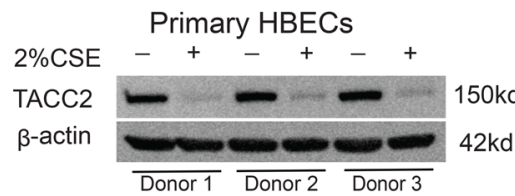

\section{Primary HAECs}

$2 \%$ CSE

TACC2

$\beta$-actin

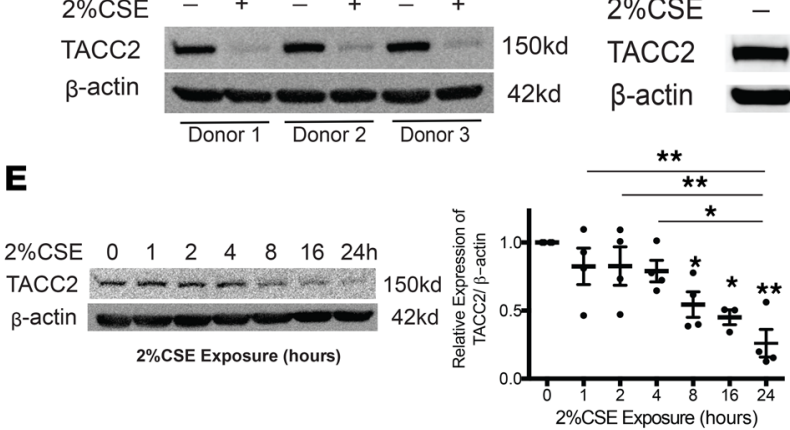

$150 \mathrm{kd}$ $42 \mathrm{kd}$

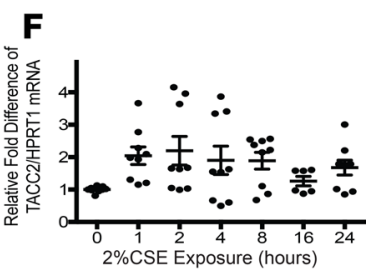

G

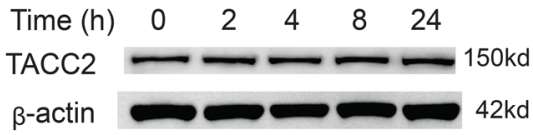

$\mathrm{CHX}+2 \% \mathrm{CSE}$

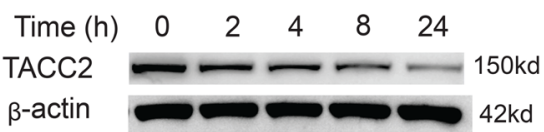

Figure 4. Cigarette smoke decreases TACC2 protein abundance in human epithelia. (A) HBEC2 cells were cultured without or with various concentrations of CSE for 24 hours. IB analysis was performed for TACC2. IB data are representative of 3 independent experiments. The relative densitometric ratios (TACC2/ $\beta$-actin) are expressed as mean $\pm \mathrm{SEM}$. ${ }^{*} P<0.05 ;{ }^{*} P<0.01$, by 1 -way ANOVA with Tukey's multiple comparisons. (B) BEAS-2B cells were treated as in A. IB analysis was performed for TACC2. IB data are representative of 3 independent experiments. (C) A549 cells were treated as in A. IB analysis was performed for TACC2. IB data are representative of 3 independent experiments. (D) Primary HBEC cells isolated from 3 nonsmoking donors and primary human alveolar epithelial cells (pHAECs) were cultured without or with $2 \%$ CSE for 24 hours. IB analysis was performed for TACC2. IB analysis to evaluate $2 \%$ CSE effects on primary HAECs is shown. (E) HBEC2 cells were cultured without or with $2 \%$ CSE for up to 24 hours. IB analysis was performed for TACC2. IB data are representative of 3 independent experiments. The relative densitometric ratios (TACC2/ $\beta$-actin) are expressed as mean $\pm \mathrm{SEM} .{ }^{*} P<0.05 ;{ }^{* *} P<0.01$. One-way ANOVA with Tukey's multiple comparisons was made. (F) Total cellular RNA was isolated, and steadystate mRNA levels of TACC2 and HPRT1 were measured. The relative fold differences (TACC2/HPRT1 mRNA) are expressed as mean \pm SEM. One-way ANOVA with Tukey's multiple comparisons was made. (G) HBEC2 cells were cultured without or with $2 \%$ CSE in the presence of $20 \mu \mathrm{g} / \mathrm{mL}$ cycloheximide (CHX) for various time periods for up to 24 hours. IB analysis was performed for TACC2. IB data are representative of 3 independent experiments.

The genetic deletion of TACC2 augments CS-induced DNA damage and emphysema. To supplement these studies with an in vivo correlate, we exposed mice with genetic deletion of TACC2. Mice 8-10 weeks of age $\left(\mathrm{Tacc}^{-/-}\right.$and $\mathrm{Tacc}^{+/+}$) were exposed to CS using 2 unfiltered cigarettes per day for the first week and 4 cigarettes per day from the second week, for a total of 6 weeks. Six weeks after the CS exposure, all mice were euthanized for lung morphometry, bronchoalveolar lavage fluid (BALF), immunoblot, and immunofluorescent image analyses. CS exposure significantly increased the alveolar chord length (CL) in $\mathrm{Tacc}^{-/-}$compared with $\mathrm{Tacc}^{+/+}$mice (Figure 7A). Furthermore, whole lung parenchymal lysates obtained from $\mathrm{Tacc}^{-1-}$ mice exhibited accumulation of the DNA damage markers (pATM and $\gamma \mathrm{H} 2 \mathrm{AX}$ ) (Figure 7B). Immunofluorescent image analysis revealed that Tacc2 $2^{-1-}$ mice exhibited a significant increase in spontaneous DNA damage $\left(\gamma \mathrm{H} 2 \mathrm{AX}\right.$ positivity) in alveolar cells relative to $\mathrm{Tacc}^{+/+}$ mice (Figure 7C). 
A

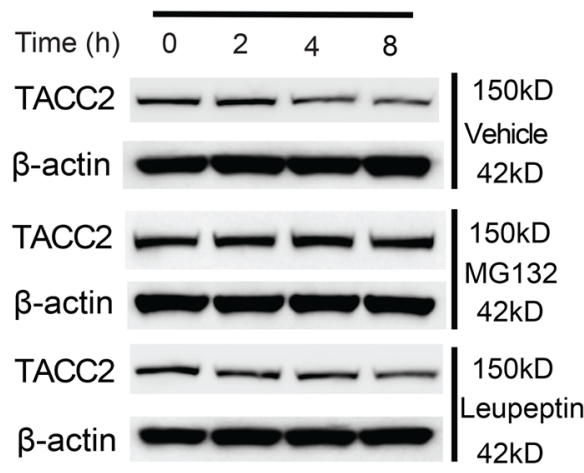

B

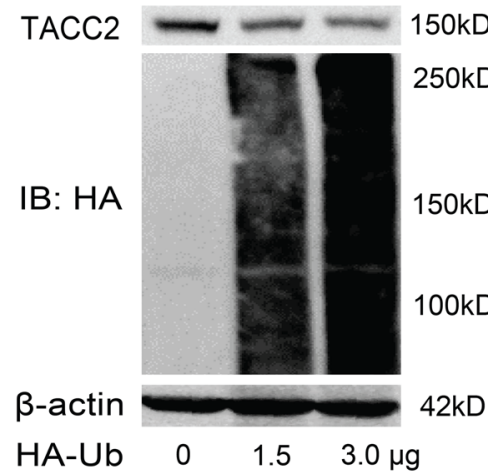

C

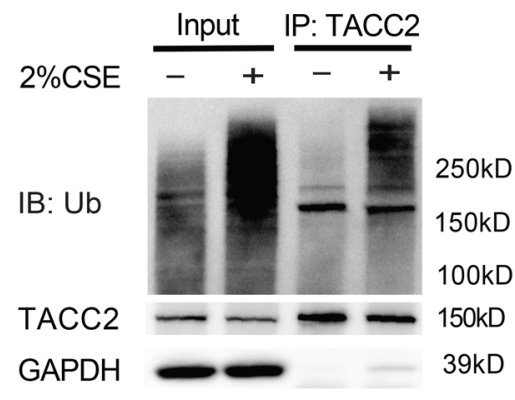

D

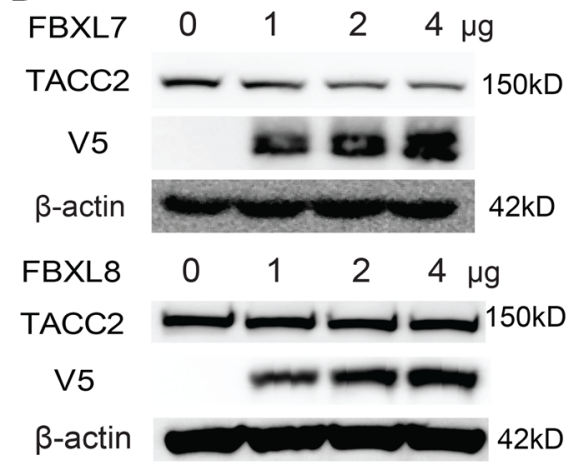

E

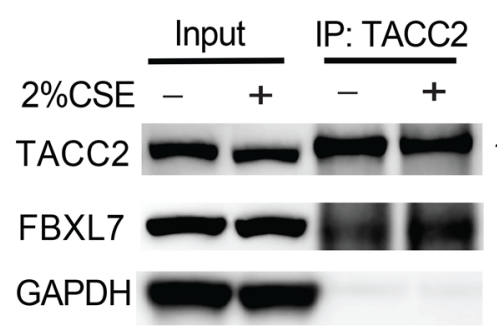

\section{$\mathbf{F}$}

G

Input

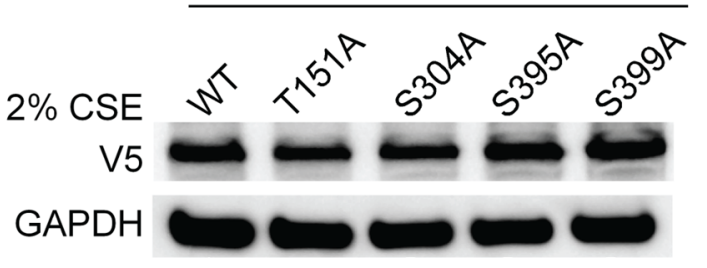

IP: V5
$150 \mathrm{kD}$

$55 \mathrm{kD}$

$39 k D$

$\begin{array}{llll}\text { FBXL7 } & - & - & +\end{array}$

E1/E2 - $\quad+\quad+$

Skp1/Cul1-Rbx1 - ++

Ubiquitin -++

V5-TACC2 +++

IB: V5

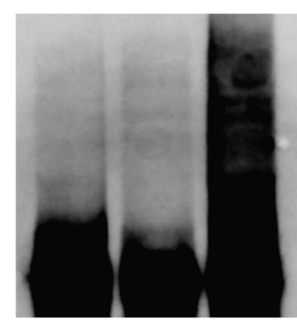

$250 \mathrm{kD}$

$150 \mathrm{kD}$

Figure 5. An SCF E3 Ligase F-box component targets TACC2 for polyubiquitination. (A) HBEC2 cells were cultured with $2 \%$ CSE and $20 \mu \mathrm{g} / \mathrm{mL}$ CHX in the presence or absence of $10 \mu \mathrm{M}$ MC132 or $10 \mu \mathrm{M}$ leupeptin for up to 8 hours. IB analysis was performed for TACC2. IB data are representative of 3 independent experiments. (B) BEAS-2B cells were transfected with the HA-ubiquitin plasmid (1.5 or $3.0 \mu$ g per well in a 6 -well plate). Cell lysates were processed for TACC2 IB (above). TACC2 was also immunoprecipitated (IP) and samples processed for HA IB (middle panel). IB data are representative of 3 independent experiments. (C) BEAS-2B cells were cultured without or with $2 \%$ CSE in the presence of $10 \mu \mathrm{M}$ MC132 for 2 hours. IP was performed with primary TACC2 antibody using cell lysates. IB analysis was performed for levels of ubiquitination (Ub), TACC2, and CAPDH. IB data are representative of 3 independent experiments. (D) BEAS-2B cells were transfected using nucleofection with increasing amounts of V5-tagged FBXL7 or FBXL8-encoding plasmids for 48 hours. IB data of TACC2 and F-box proteins are representative of 3 independent experiments. (E) BEAS-2B cells were cultured without or with $2 \%$ CSE for 4 hours. IP was performed with primary TACC2 antibody using cell lysates. IB analysis was performed for TACC2, FBXL7, and GAPDH. IB data are representative of 3 independent experiments. (F) In vitro ubiquitination of TACC2. Recombinant ubiquitin E1-activating, -E2-conjugating enzymes and ubiquitin was incubated without or with FBXL7 in the presence of V5-TACC2, and polyubiquitinylated products were detected using V5 IB. (G) BEAS-2B cells were transfected with V5-tagged TACC2 WT and phosphorylation-defective mutant (T151A, S304A, S395A, and S399A) plasmids for 48 hours and further incubated with 2\% CSE for 4 hours. IP was performed using primary V5 antibody in cell lysates. IB analysis was performed using V5, GAPDH, and FBXL7 antibodies.

CS exposure enhances epithelial cell death and lung inflammation in Tacc2 $2^{-/}$relative to Tacc2 $2^{+/+}$mice. Furthermore, both DNA damage and cell death (TUNEL positivity) were increased in alveolar epithelial cells in $\mathrm{Tacc}^{-/-}$relative to $\mathrm{Tacc}^{+/+}$mice exposed to CS (Figure 8A). BALF analysis on CS- or air-exposed mice showed that TACC2 loss increased the cell number of macrophages, lymphocytes, and neutrophils in response to CS (Figure 8B). However, the activity of neither MMP12 nor myeloperoxidase (MPO) was not significantly altered between $\mathrm{Tacc}^{+/+}$and $\mathrm{Tacc2}^{-{ }^{-1}}$ mice (Supplemental Figure 2, $\mathrm{A}$ and $\mathrm{B}$, respectively). These data suggest that the genetic deletion of TACC2 augments CS-induced lung inflammation and emphysematous changes, accompanied by increased DNA damage and cell death in alveolar epithelial cells. 

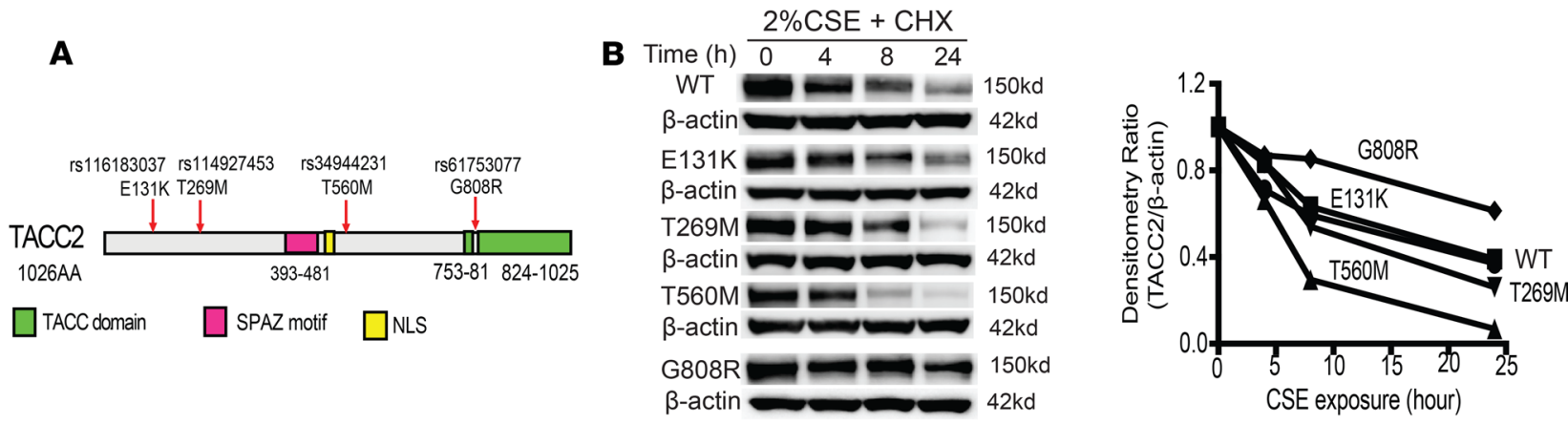

C

IB: K48

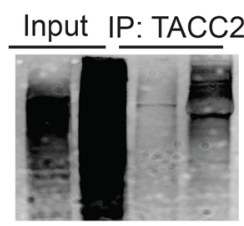

IB: K63

IB: TACC2

IB: GAPDH

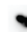

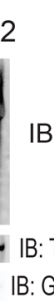

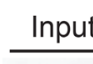

Input

IP: TACC2

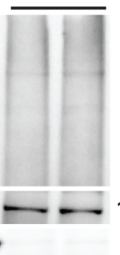

D $\frac{\text { Input }}{\text { WT }} \frac{\text { IP: V5 }}{\text { WT T560M G808R }}$

IB: V5

IB: K48

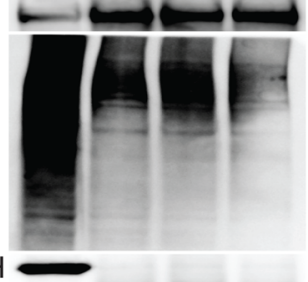

$150 k d$

WT IB: GAPDH
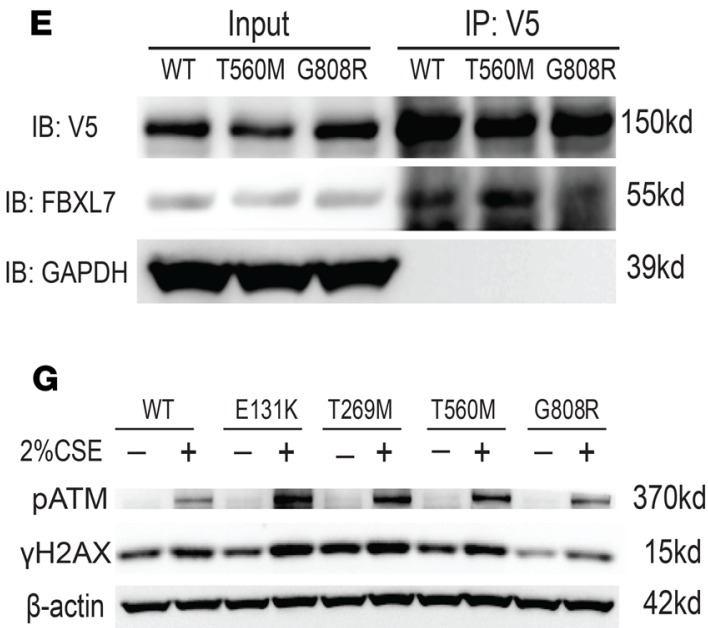

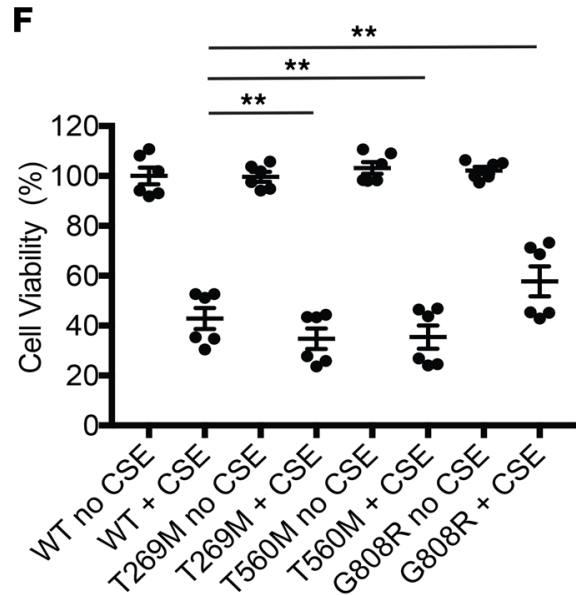

Figure 6. Naturally occurring mutations and targeted deletion of TACC2 alter functional and phenotypic responses to cigarette smoke. (A) Map illustrating rare nonsynonymous mutations of TACC2 (from the UK Biobank data) as indicated with arrows within various domains of TACC2. (B) BEAS-2B cells were transfected with V5-tagged TACC2 WT and mutant (E131K, T269M, T560M, and C808R) plasmids for 48 hours and further incubated with $2 \%$ CSE and $20 \mu \mathrm{g} / \mathrm{mL}$ CHX. The half-life of individual TACC2 mutants was monitored for up to 24 hours. IB data are representative of 3 independent experiments. The relative ratios (V5-TACC2/ $\beta$-actin) are expressed graphically. (C) BEAS-2B cells were cultured without or with $2 \%$ CSE in the presence of MC132 for 2 hours, and IP was performed using TACC2 antibody. IB analysis of the precipitates was performed using $\mathrm{K} 48$ and $\mathrm{K} 63$ antibodies. IB data are representative of 3 independent experiments. (D) BEAS2B cells were transfected with V5-tagged TACC2 WT and mutant (T560M and G808R) plasmids for 48 hours and further cultured with $2 \%$ CSE and $20 \mu \mathrm{g} / \mathrm{mL}$ CHX for 2 hours. IP was performed using primary V 5 antibody in cell lysates. IB analysis was performed using $\mathrm{K} 48$ antibody to assess levels of polyubiquitination. IB data are representative of 3 independent experiments. (E) BEAS-2B cells were treated as in D. IB analysis for FBXL7 of the immunoprecipitates (pulled down using V5 antibody) was performed to assess $F$ box protein association with TACC2 variants. IB data are representative of 3 independent experiments. (F) BEAS-2B cells were transfected with V5-tagged TACC2 WT and mutant (T269M, T560M, and G808R) plasmids for 48 hours and further cultured without or with $2 \%$ CSE for 48 hours as above. Cell viability was determined by the MTT assay. Data are expressed as mean \pm SEM for 2 independent experiments with triplicate samples (** $P<$ 0.05 vs. WT CSE-treated and individual variants) after CSE exposure. One-way ANOVA with Bonferroni correction was conducted. (C) BEAS-2B cells were treated as in $\mathbf{F}$ and harvested at 24 hours. IB analysis was performed for pATM and $\gamma \mathrm{H} 2 \mathrm{AX}$. IB data are representative of 3 independent experiments.

\section{Discussion}

Our present study shows several unique findings, including that (a) steady-state levels of TACC2 protein - but not mRNA - are substantially depleted in the lungs of smokers with severe COPD compared with smokers without COPD; (b) TACC2 depletion increases both spontaneous and CSE-induced DNA damage and cytotoxicity in cultured immortalized HBECs and suppresses HR repair, whereas TACC2 overexpression 


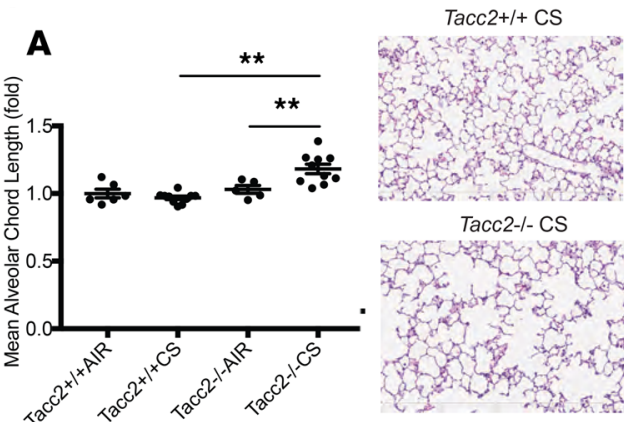

\section{B}

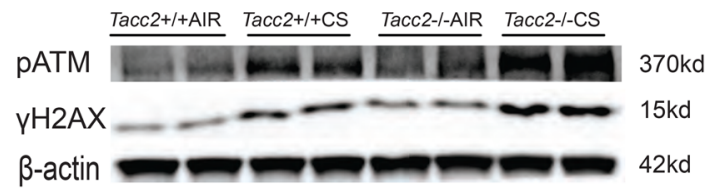

C
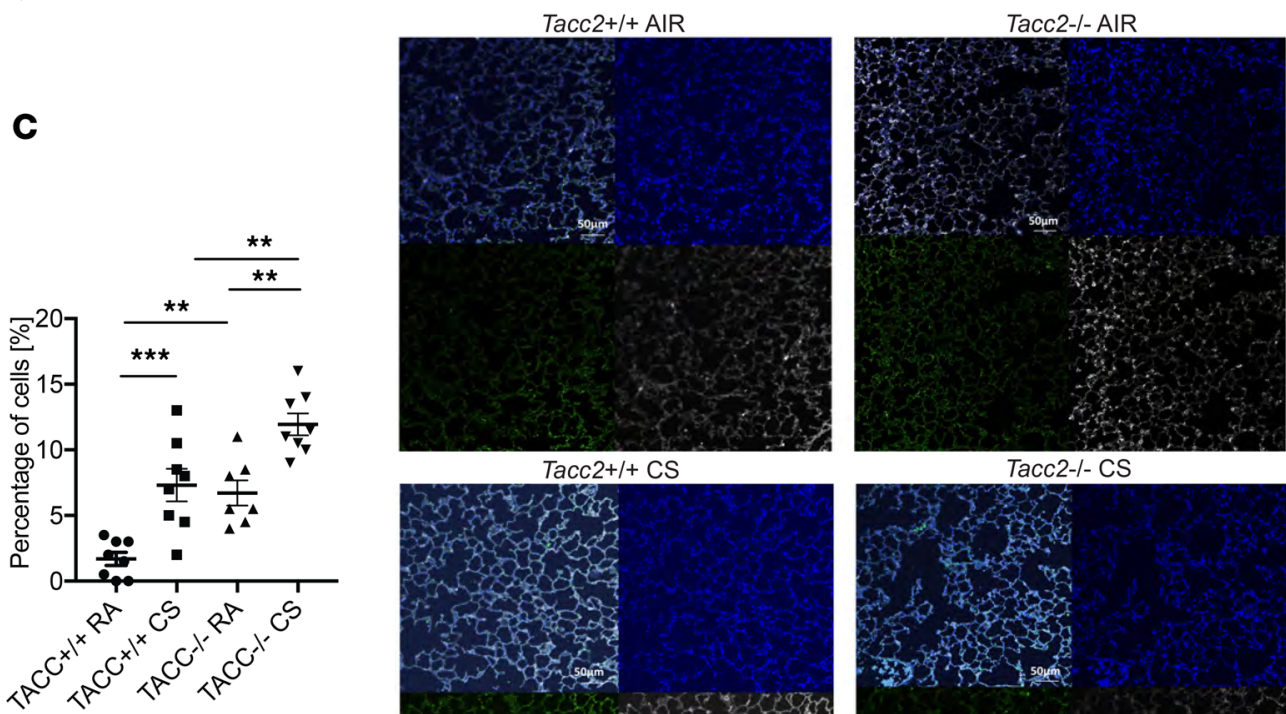

Tacc $2+1+$ CS

Tacc2-/- CS
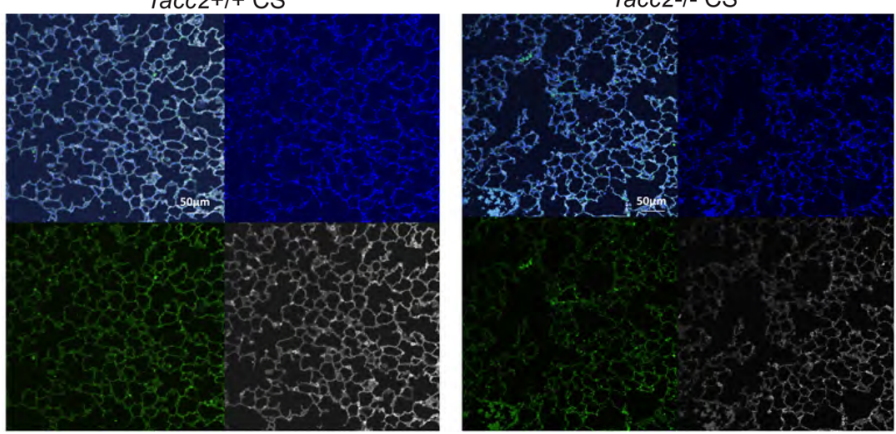

Figure 7. The genetic deletion of TACC2 augments cigarette smoke-induced DNA damage and emphysema. (A) Each group of age-matched Tacc2 ${ }^{+/+}$mice and $\operatorname{Tacc2}^{-1-}$ mice were exposed to cigarette smoke (CS) or room air (AIR) for a total of 6 weeks. Six weeks after CS or AIR exposure, all mice were euthanized. The lungs of the mice in each group were inflated under a constant pressure $(25 \mathrm{~cm} \mathrm{H} O)$ and were fixed in neutral buffered formalin, embedded in paraffin, and sectioned at $3-\mu \mathrm{m}$ thickness. Data are expressed as a relative mean chord length $(\mathrm{CL}) \pm \mathrm{SEM}$. ${ }^{*} P<0.01$. One-way ANOVA with Bonferroni correction was conducted. The mean $\mathrm{CL}$ of AIR-exposed $\mathrm{Tacc2}^{+/+}$mice was set to a value of 1.0. Representative photomicrographs of lungs from each group of CS-exposed mice processed for H\&E staining (original magnification, 200x). (B) The mice were treated as in A. Whole lung lysates were obtained from AIR- or CS-exposed Tacc $^{+/+}$and Tacc2 $2^{-1-}$ mice. IB analysis was performed for PATM and $\gamma \mathrm{H} 2 \mathrm{AX}$. (C) Analysis of $\gamma \mathrm{H} 2 \mathrm{AX}$ in alveolar epithelial cells in AIR- or CS-exposed Tacc2 ${ }^{+/+}$ and $\mathrm{Tacc}^{-1-}$ mice. Representative micrographs showing $\gamma \mathrm{H}_{2} \mathrm{AX}{ }^{+}$(green) and E-cadherin ${ }^{+}$(white) cells in peripheral lung parenchyma from AIR- or CS-exposed Tacc2 $^{+/+}$and Tacc2 $2^{-1-}$ mice. Nuclei were counterstained with DAPI (blue). Scale bar: $50 \mu \mathrm{m}$. Quantitative analysis of $\gamma \mathrm{H} 2 \mathrm{AX}{ }^{+}$alveolar epithelial cells in AIR- or CS-exposed Tacc2 $^{+/+}$and Tacc2 $^{-/-}$mice were shown as mean \pm SEM. ${ }^{* *} P<0.01 ;{ }^{* *} P<0.001$. One-way ANOVA with Bonferroni correction was conducted.

attenuates CSE effects on DNA damage and cytotoxicity; (c) mechanistically, CSE depletes TACC2 protein levels via a posttranslational mechanism that enhances TACC2 degradation through FBXL7-mediated E3 ligase activity; (d) nonsynonymous TACC2 mutations alter CSE-induced protein instability, cytotoxicity, and DNA damage in cultured HBECs; and (e) the genetic deletion of Tacc2 augments CS-induced DNA damage, cytotoxicity, and lung inflammation and produces emphysema in mice. These data raise the possibility that modulation of TACC2 to increase its cellular concentrations might serve as a novel strategy to lessen DNA damage and apoptosis in lung epithelia, thereby mitigating the severity of emphysema (Figure 9).

Accumulating evidence suggests a crucial role of apoptosis, a regulated form of cell death, of alveolar cells in the development of COPD (24). In human studies, the number of apoptotic cells as evidenced by TUNEL positivity and/or caspase- 3 activation in alveolar septal cells is increased in smokers with COPD as compared with smokers without $\operatorname{COPD}(7,25,26)$. Furthermore, apoptosis induced by active caspase- 3 
A

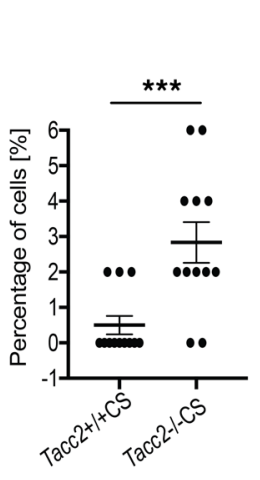

Tacc $2+/+$ CS

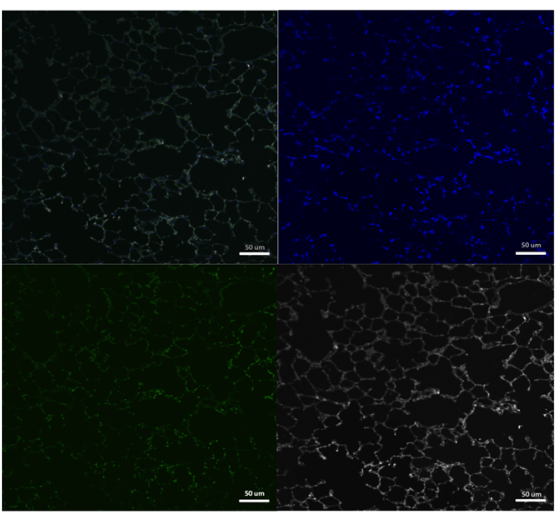

Tacc2-/- CS

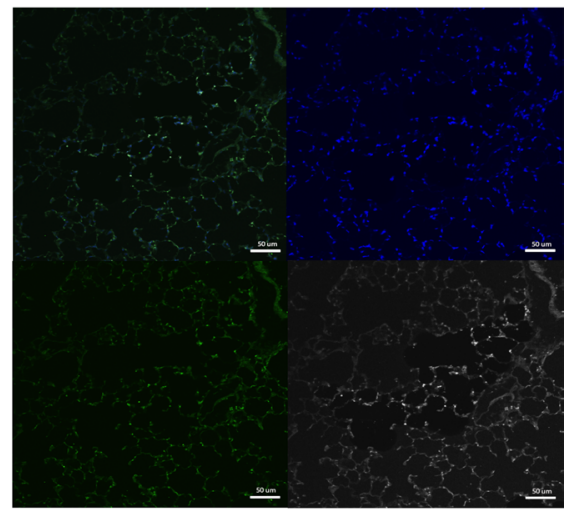

B

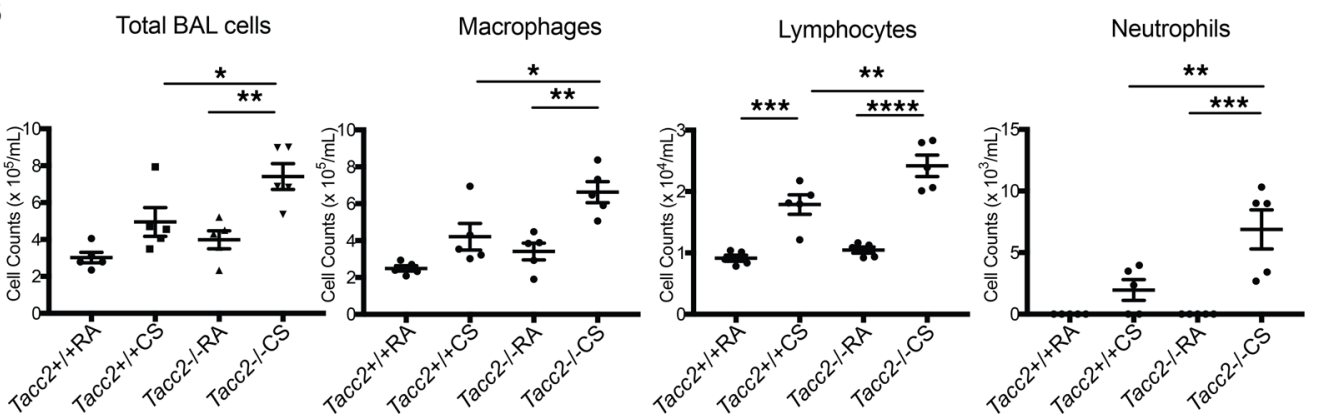

Figure 8. Cigarette smoke exposure enhances epithelial cell death and lung inflammation in Tacc2 ${ }^{-/-}$relative to Tacc2 $^{+/+}$mice. (A) Analysis of TUNEL positivity in alveolar epithelial cells in CS-exposed Tacc2 $^{+/+}$and Tacc2 $^{-1-}$ mice. Representative micrographs showing TUNEL-immunopositive cells (green) and E-cadherin (white) cells in peripheral lung parenchyma from CS-exposed $\operatorname{Tacc2}^{+/+}$and $\operatorname{Tacc}^{-1-}$ mice. Nuclei were counterstained with DAPI (blue). Scale bar: 50 $\mu \mathrm{m}$. Quantitative analysis of TUNEL ${ }^{+}$alveolar epithelial cells in CS-exposed $\operatorname{Tacc2}^{+/+}$and $\mathrm{Tacc2}^{-{ }^{--}}$mice were shown mean \pm SEM. ${ }^{* *} P<0.001$. (B) The mice were treated as in A. BAL was performed on AIR- or CS-exposed Tacc $2^{+/+}$and Tacc2 $2^{-/-}$ mice. Total BAL cell counts and differential analysis for macrophages, lymphocytes, and neutrophils were performed on isolated BAL cells. $n=5$ mice per group; data shown as mean \pm SEM. ${ }^{*} P<0.05 ;{ }^{* *} P<0.01 ;{ }^{* *} P<0.001 ;{ }^{* * *} P<0.0001$. One-way ANOVA with Bonferroni correction was conducted.

expression (27) or vascular-endothelial growth factor inhibition (28) promotes the formation of emphysema in vivo. An increase in DNA damage may contribute to apoptosis (7) because COPD lungs exhibit an increased expression of $\gamma \mathrm{H} 2 \mathrm{AX}$ (a DNA damage marker) (4). However, the molecular mechanisms of CS-induced epithelial cell apoptosis are not fully elucidated.

To date, there are no studies linking TACC2 protein expression to lung disorders. We recently identified TACC2 as a COPD candidate gene through both genomic and functional approaches. Some smokers with advanced COPD $(12.9 \%)$ carry rare deleterious mutations of the TACC2 gene, including nonsense mutation (chr10:123842508; a glutamine mutated to a stop codon) (9). In this study, we found that steady-state levels of TACC2 protein, but not mRNA, are substantially depleted in the lung of smokers with severe COPD compared with smokers without COPD. Several studies investigated a potential role of TACC2 protein in tumorogenesis $(17,29,30)$. Interestingly, the gene expression of anti-zuai-1 (AZU-1), a TACC2 splice variant, is significantly decreased in breast cancer cell lines and in primary tumors compared with nonmalignant cells and tissues (29). Furthermore, AZU-1 restoration in breast cancer cells reduces tumorigenicity both in vitro and in vivo (29). These data suggest a pretranslational mechanism for loss of this protein and support a protective role for TACC2 in neoplasia. However, Schuendeln et al. demonstrated that $\mathrm{Tacc}^{-/-}$mice do not exhibit a spontaneous clinical phenotype in Tacc2 ${ }^{-/-}$mice compared with $\mathrm{Tacc}^{+/+}$ mice (17). Further, Tacc $2^{-1-}$ murine embryonic fibroblasts (MEFs) exhibit no significant change in cell proliferation, cell cycle progression, or centrosome numbers compared with Tacc2 ${ }^{+/+} \mathrm{MEFs}$ (17). These results suggest that TACC2 is dispensable for mouse development and cell growth. However, little is known about the effects of gene-environmental interaction on cell survival and genomic stability, and a stress event could presumably modulate TACC2 cellular abundance in a given experimental context. 


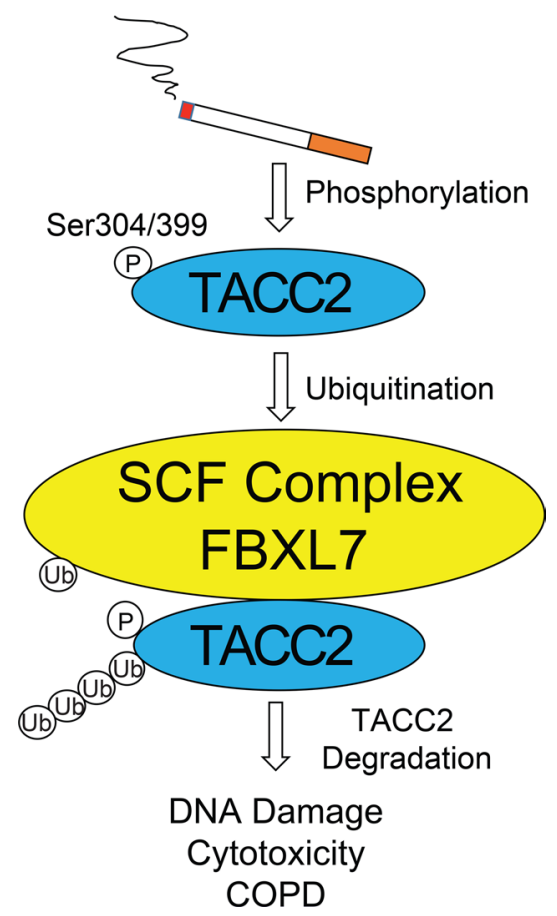

Figure 9. A proposed molecular mechanism of cigarette smoke-induced DNA damage and COPD. Cigarette smoke affects bronchial and alveolar epithelial cells, where TACC2 is destabilized by phosphorylation at Ser304/399 of TACC2 that recruits the ubiquitin E3 ligase subunit, FBXL7. Cigarette smoke-induced TACC2 degradation contributes to DNA damage, cytotoxicity, and the development of COPD.

We recently reported that suppression of TACC2 expression augments CSE-induced apoptotic cell death in immortalized HBECs (9). Here, we uncovered a unique function of TACC2 protein as a critical DNA repair factor. TACC2 protein depletion impairs the efficiency of HR repair, enhances spontaneous and CSE-induced DNA damage in vitro, and produces emphysematous changes in the lungs of CS-exposed mice. The discrepancy in the spontaneous cellular phenotype between in vitro and in vivo studies is unclear but could be due to temporal factors that allow cells to adjust to TACC2 depletion by compensatory mechanisms or underscore cell-to-cell or cell-matrix interactions that may also mask any phenotype expected in vivo. More detailed investigations to determine the molecular mechanism of TACC2-mediated DDR/repair will be required to more definitively elucidate the molecular basis for these observations in murine systems.

TACC2 protein is relatively stable, with a half-life of longer than 24 hours under native (nonstressed) conditions. However, the lifespan of TACC2 protein shortens in response to CSE via the UPS. This is a similar finding to that of Werner syndrome protein, a RecQ helicase family member and critical DNA repair enzyme that undergoes UPS-mediated degradation, an effect enhanced by CSE (31). The ubiquitin ligation is catalyzed by ligases that recognize a substrate in a relatively selective mode. Among the large family of E3 ligases, SCFs have merged as an important group of E3 complexes that regulate diverse cell signaling involved in cell cycle, cell survival, and inflammation (20,32). F-box proteins function as adaptor proteins, recruiting target proteins to the $\mathrm{E} 3$ ligase catalytic core for ubiquitination. We found that $\mathrm{SCF}^{\mathrm{FBXL} 7}$ targets TACC2 protein for polyubiquitination and the subsequent degradation in BEAS-2B cells. This result is not surprising, given the fact that FBXL7 localizes within the centrosome during spindle formation (33). However, further investigations are needed to determine the molecular recruitment mechanism of how FBXL7 gains access to TACC2 in response to CSE. This may be relevant because some posttranslational modifications, such as phosphorylation (34) or acetylation (35), are molecular recognition signatures on substrates that trigger SCF ligase recruitment to facilitate degradation. The possibility that CSE might modulate the oxidative state of residues that could enhance FBXL7 molecular interaction with this centrosomal protein is also an intriguing possibility.

A large-cohort genetic database in the UK Biobank uncovered nonsynonymous mutations of TACC2 potentially linked to emphysema (e.g., rs34944231; MAF $=0.0058 ; P=0.00038$ ). We found that the T560M mutation (rs4944231) relative to WT decreases TACC2 protein stability and cell viability, accompanied by DNA damage accumulation in CS-exposed HBECs. The G808R mutation (rs6173077), 
however, provided the opposite effects. These results are of interest, showing the loss-of-function and gain-of-function, respectively, of the molecular behavior of naturally occurring rare TACC2 variants that may modulate DNA repair processes or vulnerability to epithelial cell death associated with emphysema. Furthermore, the UK Biobank data identify several common several intronic SNPs associated with bronchiectasis (e.g., rs11200404; MAF $=0.395 ; P=3.29 \times 10^{-6}$ ). However, the molecular characterization and association of these SNPs on gene expression and phenotype of TACC2 remain to be elucidated.

Overall, these studies unveil a potentially new molecular pathway underlying COPD pathogenesis, addressing the molecular mechanisms that govern abundance of TACC2, a crucial DNA repair protein. The studies are important in that they might lead to devising FBXL7 small molecule antagonists that may lay the groundwork for pathway validation and preclinical testing of such therapeutic strategies for smoking-induced COPD.

\section{Methods}

Human lung tissue samples. Frozen lung parenchymal tissue obtained from smokers without $(n=6)$, with moderate $(n=6)$, or with severe/very severe COPD $(n=10)$ were provided by the Lovelace Respiratory Research Institute (LRRI, Albuquerque, New Mexico, USA) and Lung Tissue Research Consortium (LTRC, Bethesda, Maryland, USA). Fresh lung parenchymal tissue obtained from 3 nonsmokers without known preexisting lung disease were provided by the University of Pittsburgh Tissue Culture Core.

Single cell RNA sequencing. Single cell RNA sequencing was conducted using lung parenchymal tissues obtained from 3 normal human subjects and 3 severe COPD patients. Peripheral lung tissues were enzymatically digested to achieve a single cell suspension. Mononuclear cells were then separated into minireaction partitions, or Gel Bead-in-EMulsion (GEMs) formed by oil microdroplets, each containing a gel bead and a cell, using a Chromium instrument (10× Genomics). The reaction mixture/emulsion with captured and barcoded mRNAs were removed from the Chromium instrument, followed by reverse transcription. The cDNA samples were fragmented and amplified. The libraries were then constructed, purified, and quantified. Libraries were sequenced on an Illumina NextSeq 500. Alignment to the human reference genome GRCh38 and the generation of a cell $\times$ gene count matrix was performed using the pipeline Cell Ranger developed by $10 \times$ Genomics. Two-dimensional t-SNE plots were generated based on global gene expression relationships.

Reagents and antibodies. Polyvinylidene difluoride (PVDF, catalog 1620177) membranes were obtained from Bio-Rad. ECL Plus was obtained from Amersham (catalog K-12043-D20). The protein synthesis inhibitor cycloheximide (CHX, catalog 66-81-9), the protease inhibitor leupeptin (catalog L5793), the proteasome inhibitor MG132 (catalog 133407-82-6), GAPDH (catalog G9545), $\beta$-actin (catalog A4700), and horseradish peroxidase-conjugated anti-rabbit (catalog A1949) or anti-mouse Ig (catalog A3682) antibodies were purchased from Sigma-Aldrich. Antibodies were obtained from various sources: TACC2 was from Origene (catalog TA804145); HA-TAG (catalog 3724S), antibodies and phosphorylation-specific antibody for ATM (catalog 13050), and H2AX (catalog 2577) were from Cell Signaling Technology; HPRT was from Abcam (catalog ab109021); TACC2 CRISPR/Cas9 plasmid (catalog sc-405864-NIC-2) and FBXL7 (catalog sc-374319) antibodies were obtained from Santa Cruz Biotechnology Inc. Immobilized protein A/G beads (catalog 20421), V5 (catalog PA1-993), the pcDNA3.1D-V5-His-TOPO cloning kit (catalog 45-0158), and TOP10 competent cells (catalog C404010) were purchased from Thermo Fisher Scientific. Tissue culture plates (catalog 353043) were obtained from Corning.

Preparation of CSE. Research cigarettes (3R4F) were purchased from the University of Kentucky (Lexington, Kentucky, USA). CS exposure solutions were prepared as previously described (31).

Cell culture. Both pHBECs and pHAECs were isolated from nonsmokers and maintained under a protocol approved by the University Pittsburgh IRB. HBEC2 cells (immortalized HBECs) originally generated by Ramirez et al. (22), and BEAS-2B (CRL9609) and A549 cells (CCL185) purchased from America Type Culture Collection, were cultured and maintained as previously described $(36,37)$. Experiments were performed in 6-well Costar tissue culture plates or p100 dishes $(100 \mathrm{~mm})$ at a starting cell density of $1.5 \times$ $10^{3} / \mathrm{cm}^{2}$. Cell counts were generated using an electric particle counter (Beckman Coulter, catalog TC20). Twenty-four hours after plating, cells were exposed to various concentrations of CSE for designated hours.

Immunofluorescence analysis. Following CSE exposure, the cells were washed with PBS and fixed by 4\% PFA (Thermo Fisher Scientific, catalog AAJ19943K2) for 15 minutes. Cells were washed with PBS 3 times and permeabilized with $0.2 \%$ Triton X-100 (Thermo Fisher Scientific, catalog BP151) in PBS for 8 minutes. Cells were washed with PBS 3 times and blocked with 5\% BSA (RPI, catalog A30075) in PBS-T (PBS+0.1\% Tween 20 [Sigma-Aldrich, catalog P1379]) for 0.5 hours and then were incubated with primary 
antibodies (TACC2, 1:200; rabbit $\gamma \mathrm{H} 2 \mathrm{AX}, 1: 400)$ at $4^{\circ} \mathrm{C}$ overnight. After being washed 3 times with PBS-T, cells were incubated with secondary antibodies for 1 hour (Alexa Fluor anti-mouse 488 [Invitrogen, catalog A21202)] and anti-rabbit 647 [Invitrogen, catalog A21244], 1: 500) at room temperature. After being washed with PBS-T, cells were incubated with DAPI (Thermo Fisher Scientific, catalog D1306) for 10 minutes and washed with PBS-T 3 times.

HR assay. DR-GFP-stably expressing HeLa cells were incubated in DMEM without sodium pyruvate with 10\% FBS and 1\% GlutaMAX (Thermo Fisher Scientific, catalog 10566-016). Cells were transfected with $20 \mu \mathrm{M}$ siCtrl or siTACC2 using RNAiMax (Thermo Fisher Scientific, catalog 13778150) for 24 hours. Media was changed, and cells were transfected with pCMV-ISce-I plasmid using Lipofectamine 2000 (Thermo Fisher Scientific, catalog 11668019) for 48 hours and kept in the dark. Then, cells were spun at $600 \mathrm{rpm}$, washed with $1 \times$ PBS, and analyzed by a fluorescence activated cell sorting (FACS).

Immunoblot analysis and IP. Cell lysates were prepared in RIPA buffer (Sigma-Aldrich, catalog R0218) with protease inhibitors (Thermo Fisher Scientific, catalog A32961) and analyzed by immunoblotting as previously described (38). For immunoprecipitation, about $1 \mathrm{mg}$ cell lysates were incubated with specific primary antibody overnight and were then added to $40 \mu \mathrm{L}$ protein A/G agarose beads (Thermo Fisher Scientific, catalog 78609) for an additional 2 hours at room temperature. The precipitation complex was washed by cold PBS-T (PBS+ $0.1 \%$ Tween 20) three times for 5 minutes each. The precipitation was added to $50 \mu \mathrm{L}$ lysis buffer and analyzed by immunoblotting with an enhanced chemiluminescence detection system (Advansta, catalog K-12043-D20). Equal loading of protein samples from each group was evaluated using $\beta$-actin antibody or GAPDH after using Restore WB stripping buffer (Thermo Fisher Scientific, catalog 21059).

Cell fractionation. After being cultured with or without 2\% CSE for 2 hours, HBEC2 cells were washed with cold PBS and digested by trypsin-EDTA (Thermo Fisher Scientific, catalog 25200056). Cytoplasmic and nuclear fractions were separated using a cell fractionation kit (Cell Signaling Technology, catalog 9038) according to the manufactural instructions.

Flow cytometric analysis. Flow cytometry with dual staining for Annexin V (BioLegend, catalog 640906) and H2AX was performed as previously described with some modifications (9). Briefly, after being harvested with trypsinization, 105 cells were stained in $1 \times$ binding buffer $(0.01 \mathrm{M}$ HEPES, pH 7.4; $0.14 \mathrm{M} \mathrm{NaCl} ; 0.25 \mathrm{mM} \mathrm{CaCl2}$ ) using $5 \mu \mathrm{L}$ of Annexin V-FITC and $10 \mu \mathrm{L}$ H2AX-PerCPCy5.5 (BD Biosciences, catalog 564718). The cells were then incubated in the dark for 15 minutes at room temperature, and the percentage of FITC- and PerCP-Cy5.5-positive cells were quantified using FACS; data were analyzed using FlowJo software (version 7.6.3).

CRISPR/Cas9. BEAS-2B cells were transfected with double nickase plasmids (sc-405864-NIC-2) purchased from Santa Cruz Biotechnology Inc. according to the manufactural instructions. After puromycin selection at $1 \mu \mathrm{g} / \mathrm{mL}$ for 72 hours, more than 20 clones were isolated to confirm successful $\mathrm{KO}$ of TACC2.

Site-directed mutagenesis. QuickChange II XL site-directed mutagenesis kit (Agilent, catalog 200521) was used to introduce mutagenesis according to the manufactural instructions. Using the primer pair of T151A, S304A, S395A, and S399A (Table 2), mutant strands were synthesized by PCR. Using the primer pair of E131K, T269M, T560M, and G808R (Table 2), mutant strands were synthesized by PCR. The PCR product was treated with Dpn I to digest the parental DNA template and transformed into XL1-Blue supercompetent cells. The mutation was verified by DNA sequencing.

$R N A$ isolation and quantitative PCR. RNA was isolated from lung tissue samples using the RNeasy mini kit (Qiagen, catalog 74104). Quantitative RCR (qPCR) analysis for TACC2 mRNA was performed using Taqman One-Step RT-PCR Master Mix Reagents (Thermo Fisher Scientific, catalog A15299) as previously described (39). The following sets of probes TACC2 (Hs00610617_m1) and HPRT1 (Hs02800695_m1) were purchased from Applied Biosystems. Reverse transcription PCR reactions were performed using a real-time ABI PRISM 7900HT PCR system. HPRT1 mRNA levels were used as control to standardize TACC2 mRNA expression.

Construction of plasmids and cell transfection. The open reading frame of the human TACC 2 was amplified by PCR using the forward primer 5'-CACCATGGCCAACTGTGAGCGTAC-3' and the reverse primer 5'-GATCCAGTTCTGAGCACAG-3'. The PCR product was purified from an agarose gel, followed by cloning into a pcDNA3.1D-V5-His plasmid. Cells were transfected with $2 \mu \mathrm{g}$ plasmid in $100 \mu \mathrm{L}$ HEPES (20 mM in PBS) using electroporation executed with a nuclear transfection apparatus (Amaxa Biosystems) with a preset program T-013. After 48 hours, cells were harvested for further analysis. 
Table 2. Primers used for TACC2 mutagenesis

\begin{tabular}{|c|c|}
\hline Primers & Sequences \\
\hline T151A-F & 5'- CACGGAGGAAGTCCGCGGATTCCGTCCCCATC -3' \\
\hline T151A-R & 5'- GATGGGGACGGAATCCGCGGACTTCCTCCGTG -3' \\
\hline S304A-R & 5'- CCTCCCGACAGGGGGTGCGTTCTGCACTCTGCC -3' \\
\hline S395A-F & 5'- CAACCAGCAGGAAAACCCGCCTCCTACCAAAAAGATAGG -3' \\
\hline S395A-R & 5'- CCTATCTTTTTGGTAGGAGGCGGGTTTTCCTGCTGGTTG -3’ \\
\hline E131K-F & 5'- GATACCTTTCAGACCTTCAAGCCTCGTCCCTCAGACGC -3' \\
\hline E131K-R & 5'- GCGTCTGAGGCACGAGGCTTCAAGGTCTGAAAGGTATC -3' \\
\hline T269M-F & 5'- CCAGCCTTATTGGAGGAGATGCCCCTTGAGCCCGCTG -3' \\
\hline T269M-R & 5'-CAGCGGGCTCAAGGGGCATCTCCTCCAATAAGGCTGG -3' \\
\hline T560M-F & 5'-CACCAACCAGAAGTGGATGTGCATGACAGTGGACC -3' \\
\hline
\end{tabular}

Animals. Tacc2 ${ }^{+/-}$mice (C57B1/6 background) were provided by James Ihle's lab (17).

CS exposure. Mice were exposed to 6 weeks of either CS (4 cigarettes/day, 5 days/week, $n=10$ for $\mathrm{Tacc}^{+/+}$and $\mathrm{Tacc}^{2^{-/}}$mice) or room $\operatorname{air}(\mathrm{AIR})\left(n=5\right.$ for $\mathrm{Tacc}^{2^{+/+}}$mice; $n=6$ for $\mathrm{Tacc}^{-/-}$mice), beginning at 9-10 weeks of age, as previously described (40). Research cigarettes (3R4F) were purchased from the University of Kentucky.

Lung histology. After completing CS exposure, mice were euthanized. The lungs of mice were fixed under a constant pressure $\left(25 \mathrm{~cm} \mathrm{H}_{2} \mathrm{O}\right)$ in $10 \%$ neutral buffered formalin (Thermo Fisher Scientific, catalog 245684)for 2 hours. After fixation for 24 hours in formalin, lungs were embedded in paraffin and sectioned at $3-\mu \mathrm{m}$ thickness as previously described (41). Using Scion Image software (Version 4.0.2), mean alveolar CL was measured on 10 randomly selected $200 \times$ fields per slide as previously described (40).

$B A L F$ collection and analysis. After mice were euthanized following CS or AIR exposure for 6 weeks $(n$ $=5$ per group for $\mathrm{Tacc}^{+/+}$and $\mathrm{Tacc}^{-/-}$mice), the mouse lungs were lavaged 3 times with $0.5 \mathrm{~mL}$ of PBS containing $2 \%$ BSA. Total cell numbers in BALF were counted, and cytospins were prepared $\left(1 \times 10^{5}\right.$ cells $/$ slide) and stained with the Kwik-DiFF Stain Kit (Thermo Fisher Scientific, catalog 9990700). Cell differential counts were performed to quantify inflammatory cells numbers.

Immunofluorescent staining. Mouse lung tissue sections were deparaffinized and rehydrated using xylene, followed by graded ethanol washes and deionized water. Heat-induced epitope retrieval in $10 \mathrm{mM}$ sodium citrate buffer ( $\mathrm{pH}$ 6.0) was used, and endogenous peroxidase activity was quenched in $3 \% \mathrm{H}_{2} \mathrm{O}_{2}$. Sections were blocked and incubated overnight at $4^{\circ} \mathrm{C}$ with anti- $\gamma \mathrm{H} 2 \mathrm{AX}$ rabbit primary antibody (Cell Signaling Technology, catalog 2577). Slides were washed using PBS with $0.4 \%$ Triton X-100 and incubated with anti-rabbit Alexa Fluor 488 secondary antibody (Invitrogen, Cat\# A21206) for 1 hour at room temperature. Slides were then washed using PBS-T and incubated with Mouse E-cadherin (Cell Signaling Technology, catalog 14472) for 1 hours at room temperature. Sections were washed in PBS-T and incubated with anti-mouse Alexa Fluor 647 secondary (Invitrogen). Nuclei were stained with DAPI. Images were acquired using confocal microscope followed by image analysis using ImageJ software (NIH).

TUNEL assay. Mouse lungs were harvested, fixed in 10\% formalin for 48 hours, embedded in paraffin, and sectioned. After removal of the paraffin, slides were deparaffinized, hydrated, and permeabilized with $0.1 \%$ Triton X-100 in $0.1 \%$ citrate buffer ( $\mathrm{pH}$ 6.0). An In Situ Cell Death Detection Kit (Sigma-Aldrich, catalog 11684795910) was performed on these slides according to the manufacturer's instructions. After staining, the sections were visualized under a confocal microscope (NIKON A1 Spectral Confocal) using an excitation wavelength in the range of $450-500 \mathrm{~nm}$ and detection in the range of $515-565 \mathrm{~nm}$. 
Immunofluorescent analysis. Twelve representative images obtained using confocal microscopy from each group were randomly selected (4 mice/group, 3 pictures/mouse). Images were next adjusted to $256 \times 256$ pixels. The manual count of the number of basal epithelial cells within the regions of interest was performed twice for a focal area by the same trained person. To check the correctness of counting after this step, images were uploaded to ImageJ software (NIH) and automatically counted using an image-based tool for counting nuclei. The results were put into GraphPad software, and the results were shown as a percentage of positively stained cells (TUNEL and $\gamma \mathrm{H} 2 \mathrm{AX}$ ) compared with all the captured cells.

MMP-12 assay. SensoLyte 520 MMP-12 assay kit (AnaSpec, catalog AS-71157) was used to measure MMP-12 activity according to the manufacturer's instructions. After completing CS or AIR exposure, the lungs of mice were harvested and homogenized in assay buffer. The samples were incubated for 2 hours at $37^{\circ} \mathrm{C}$ in the assay buffer with $1 \mathrm{mM}$ APMA (4-aminophenylmercuric acetate). MMP-12-containing samples and substrate solution were added into 96-well plates. Fluorescence intensity was measured at $\mathrm{Ex} / \mathrm{Em}=490 / 520 \mathrm{~nm}$.

MPO assay. MPO antigen levels in BAL and lungs tissues lysates were measured using the murine MPO ELISA kits (Hycult Biotechnology, catalog HK210) according to the manufacturer's instructions. Briefly, samples and standards were applied in a volume of $100 \mu \mathrm{L}$ in duplicates onto 96-well plates precoated with capture antibody. After 60 minutes and washing 4 times, biotinylated tracer antibody was added to each well. After the incubation for 1 hour for binding of biotin to streptavidin-peroxidase conjugate, the color development with tetramethylbenzidine was performed for 30 minutes. The color reaction was stopped by the addition of Stop Solution. Color intensity was measured by determination of the absorbance at $450 \mathrm{~nm}$ using an ELISA plate reader. The mouse MPO level of each sample was calculated from the standard curve with concentrations between 1.6 and $100 \mathrm{ng} / \mathrm{mL}$ of mouse MPO by serial diluted standards.

Statistics. Student unpaired 2-tailed $t$ test was used for the comparison of 2 groups. For multiple comparisons, a 1-way ANOVA with Bonferroni correction with post hoc comparisons were used. Data were expressed as mean \pm SEM, and $P<0.05$ was considered statistically significant.

Study approval. The use of human subject samples was approved by New Mexico VA Healthcare System and University of Pittsburgh IRB. All animal experiments were approved by the IACUC and were performed at New Mexico VA Healthcare System, VA Pittsburgh Healthcare System, and University of Pittsburgh, approved by the Association for the Assessment and Accreditation for Laboratory Animal Care International.

\section{Author contributions}

XL, JHJ, LL, TN, and RKM conceived and designed the experiments. XL, JHJ, AH, TC, JS, MR, LL, SW, YT, PS, and TK performed the experiments. LL, FS, RKM, TK, RL, and TN analyzed the data. YZ, CZ, PS, DC, and RKM contributed reagents/materials/analysis tools. XL, JHJ, RKM, and TN wrote and edited the paper.

\section{Acknowledgments}

This manuscript was supported, in part, by the US Department of Veterans Affairs, Veterans Health Administration, Office of Research and Development, Biomedical Laboratory Research, and Development by a Merit Review award CX001048 (TN) and CX000105 (RKM and TN). This work was also supported by American Heart Association (TN); NIH grants R01HL149719 to TN; 01UH3HL123502, R01HL096376, R01HL097376, R01HL098174, R01HL081784, and P01HL114453 to RKM; and R01HL128297 and R01HL141080 to PS. Frozen lung parenchymal tissue samples from smokers without COPD and with severe COPD were provided by LTRC. Frozen lung parenchymal lung tissue from smokers with moderate COPD were provided by LRRI. Tacc $2^{-/-}$mice were provided by James N. Ihle.

Address correspondence to: Toru Nyunoya, University of Pittsburgh, NW628 UPMC Montefiore, 3459 Fifth Avenue, Pittsburgh, Pennsylvania 15261, USA. Phone: 412.624.7280; Email: nyunoyat@upmc.edu. 
1. Straus SE, McAlister FA, Sackett DL, Deeks JJ. The accuracy of patient history, wheezing, and laryngeal measurements in diagnosing obstructive airway disease. CARE-COAD1 Group. Clinical Assessment of the Reliability of the Examination-Chronic Obstructive Airways Disease. JAMA. 2000;283(14):1853-1857.

2. Han MK. Update in chronic obstructive pulmonary disease in 2010. Am J Respir Crit Care Med. 2011;183(10):1311-1315

3. Ito K, Barnes PJ. COPD as a disease of accelerated lung aging. Chest. 2009;135(1):173-180.

4. Shi Y, et al. Retinoic acid-related orphan receptor- $\alpha$ is induced in the setting of DNA damage and promotes pulmonary emphysema. Am J Respir Crit Care Med. 2012;186(5):412-419.

5. Jackson SP, Bartek J. The DNA-damage response in human biology and disease. Nature. 2009;461(7267):1071-1078

6. Chapman JR, Taylor MR, Boulton SJ. Playing the end game: DNA double-strand break repair pathway choice. Mol Cell. 2012;47(4):497-510.

7. Aoshiba K, Zhou F, Tsuji T, Nagai A. DNA damage as a molecular link in the pathogenesis of COPD in smokers. Eur Respir J. 2012;39(6):1368-1376.

8. Volonte D, Kahkonen B, Shapiro S, Di Y, Galbiati F. Caveolin-1 expression is required for the development of pulmonary emphysema through activation of the ATM-p53-p21 pathway. J Biol Chem. 2009;284(9):5462-5466.

9. Bruse $\mathrm{S}$, et al. Whole exome sequencing identifies novel candidate genes that modify chronic obstructive pulmonary disease susceptibility. Hum Genomics. 2016;10:1.

10. Peset I, Vernos I. The TACC proteins: TACC-ling microtubule dynamics and centrosome function. Trends Cell Biol. 2008;18(8):379-388

11. Rose A, Schraegle SJ, Stahlberg EA, Meier I. Coiled-coil protein composition of 22 proteomes--differences and common themes in subcellular infrastructure and traffic control. BMC Evol Biol. 2005;5:66.

12. Lee MJ, Gergely F, Jeffers K, Peak-Chew SY, Raff JW. Msps/XMAP215 interacts with the centrosomal protein D-TACC to regulate microtubule behaviour. Nat Cell Biol. 2001;3(7):643-649.

13. Gergely F, Kidd D, Jeffers K, Wakefield JG, Raff JW. D-TACC: a novel centrosomal protein required for normal spindle function in the early Drosophila embryo. EMBO J. 2000;19(2):241-252.

14. Lauffart B, Gangisetty O, Still IH. Molecular cloning, genomic structure and interactions of the putative breast tumor suppressor TACC2. Genomics. 2003;81(2):192-201.

15. Ha GH, Kim JL, Breuer EK. Transforming acidic coiled-coil proteins (TACCs) in human cancer. Cancer Lett. 2013;336(1):24-33.

16. Raff JW. Centrosomes and cancer: lessons from a TACC. Trends Cell Biol. 2002;12(5):222-225.

17. Schuendeln MM, et al. The centrosomal, putative tumor suppressor protein TACC2 is dispensable for normal development, and deficiency does not lead to cancer. Mol Cell Biol. 2004;24(14):6403-6409.

18. Hochstrasser M. Biochemistry. All in the ubiquitin family. Science. 2000;289(5479):563-564.

19. Tyers M, Willems AR. One ring to rule a superfamily of E3 ubiquitin ligases. Science. 1999;284(5414):601-604.

20. Cenciarelli C, Chiaur DS, Guardavaccaro D, Parks W, Vidal M, Pagano M. Identification of a family of human F-box proteins. Curr Biol. 1999;9(20):1177-1179.

21. Min T, Bodas M, Mazur S, Vij N. Critical role of proteostasis-imbalance in pathogenesis of COPD and severe emphysema. J Mol Med. 2011;89(6):577-593.

22. Ramirez RD, et al. Immortalization of human bronchial epithelial cells in the absence of viral oncoproteins. Cancer Res. 2004;64(24):9027-9034.

23. Beli P, et al. Proteomic investigations reveal a role for RNA processing factor THRAP3 in the DNA damage response. Mol Cell. 2012;46(2):212-225.

24. Park JW, Ryter SW, Choi AM. Functional significance of apoptosis in chronic obstructive pulmonary disease. COPD. 2007;4(4):347-353.

25. Yokohori N, Aoshiba K, Nagai A, Respiratory Failure Research Group in Japan. Increased levels of cell death and proliferation in alveolar wall cells in patients with pulmonary emphysema. Chest. 2004;125(2):626-632.

26. Imai K, Mercer BA, Schulman LL, Sonett JR, D'Armiento JM. Correlation of lung surface area to apoptosis and proliferation in human emphysema. Eur Respir J. 2005;25(2):250-258.

27. Aoshiba K, Yokohori N, Nagai A. Alveolar wall apoptosis causes lung destruction and emphysematous changes. Am J Respir Cell Mol Biol. 2003;28(5):555-562.

28. Petrache I, et al. Ceramide upregulation causes pulmonary cell apoptosis and emphysema-like disease in mice. Nat Med. 2005;11(5):491-498.

29. Chen HM, Schmeichel KL, Mian IS, Lelièvre S, Petersen OW, Bissell MJ. AZU-1: a candidate breast tumor suppressor and biomarker for tumor progression. Mol Biol Cell. 2000;11(4):1357-1367.

30. Onodera Y, et al. TACC2 (transforming acidic coiled-coil protein 2) in breast carcinoma as a potent prognostic predictor associated with cell proliferation. Cancer Med. 2016;5(8):1973-1982.

31. Nyunoya T, et al. Cigarette smoke induces cellular senescence via Werner's syndrome protein down-regulation. Am J Respir Crit Care Med. 2009;179(4):279-287.

32. Ilyin GP, Rialland M, Glaise D, Guguen-Guillouzo C. Identification of a novel Skp2-like mammalian protein containing F-box and leucine-rich repeats. FEBS Lett. 1999;459(1):75-79.

33. Coon TA, Glasser JR, Mallampalli RK, Chen BB. Novel E3 ligase component FBXL7 ubiquitinates and degrades Aurora A, causing mitotic arrest. Cell Cycle. 2012;11(4):721-729.

34. Ravid T, Hochstrasser M. Diversity of degradation signals in the ubiquitin-proteasome system. Nat Rev Mol Cell Biol. 2008;9(9):679-690.

35. Zou C, Mallampalli RK. Regulation of histone modifying enzymes by the ubiquitin-proteasome system. Biochim Biophys Acta. 2014;1843(4):694-702.

36. Wang Q, et al. RIP1 potentiates BPDE-induced transformation in human bronchial epithelial cells through catalase-mediated suppression of excessive reactive oxygen species. Carcinogenesis. 2013;34(9):2119-2128.

37. Xu J, et al. Autophagy-Mediated Degradation of IAPs and c-FLIP(L) Potentiates Apoptosis Induced by Combination of TRAIL and Chal-24. J Cell Biochem. 2016;117(5):1136-1144.

38. Jang $\mathrm{JH}$, et al. Aldehyde dehydrogenase 3A1 protects airway epithelial cells from cigarette smoke-induced DNA damage and 
cytotoxicity. Free Radic Biol Med. 2014;68:80-86.

39. Schwalm K, et al. Expression of the proapoptotic protein Bax is reduced in bronchial mucous cells of asthmatic subjects. Am J Physiol Lung Cell Mol Physiol. 2008;294(6):L1102-L1109.

40. Radder JE, et al. Extreme Trait Whole-Genome Sequencing Identifies PTPRO as a Novel Candidate Gene in Emphysema with Severe Airflow Obstruction. Am J Respir Crit Care Med. 2017;196(2):159-171.

41. Nyunoya T, March TH, Tesfaigzi Y, Seagrave J. Antioxidant diet protects against emphysema, but increases mortality in cigarette smoke-exposed mice. COPD. 2011;8(5):362-368.

42. Rabe KF, et al. Global strategy for the diagnosis, management, and prevention of chronic obstructive pulmonary disease: GOLD executive summary. Am J Respir Crit Care Med. 2007;176(6):532-555. 